\title{
Kinematics and stellar content of the Milky Way populations toward the North Galactic Pole ${ }^{\star}$
}

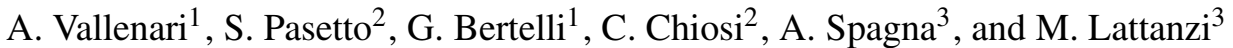

1 INAF, Padova Astronomical Observatory, vicolo Osservatorio 5, 35122, Padova,Italy

e-mail: [pasetto; vallenari; bertelli]@pd.astro.it

2 Department of Astronomy, Padova University, vicolo dell'Osservatorio 2, 35122, Padova, Italy e-mail: chiosi@pd.astro.it

3 INAF, Torino Astronomical Observatory, via Osservatorio 20, 10025 Pino Torinese, Italy

e-mail: spagna@to.astro.it

Received 16 December 2005 / Accepted 17 January 2006

\section{ABSTRACT}

Aims. The formation and evolution of galaxies is one of the forefront problems of Astrophysics. Detailed studies of our own Galaxy are the first step to understand these complex processes. In this paper we discuss the stellar populations and kinematics toward the North Galactic Pole (NGP) using data taken from the Guide Star Catalog II.

Methods. The Padova Galaxy model has been upgraded including the possibility of simulating in a consistent way color magnitude diagrams, luminosity functions and proper motions. Particular care is paid to simulate the kinematics of the thin disk, using a velocity dispersion tensor with off-diagonal term accounting for the vertical tilt.

Results. To reproduce the observational color distribution of the studied fields, one of the most critical parameters is the IMF slope. The canonical Kroupa IMF gives a poor fit of the data. The most convincing solutions are obtained assuming that about $40 \%$ of the stars are in binaries and that the initial mass function changes slope at $0.8 M_{\odot}$ instead than at the canonical value of $0.5 M_{\odot}$. We derive a Thin Disk IMF slope of $\alpha=0.9 \pm 0.2$ in the mass range $0.2-0.8 M_{\odot}$. We derive the velocity ellipsoids of the Thin disk populations. The Thick disk and the Thin disk are well separated concerning their kinematic properties. The Thick disk is found to have a mean rotational velocity of $\overline{v_{\mathrm{c}}}=178 \pm 8 \mathrm{~km} \mathrm{~s}^{-1}$ implying a moderate rotational lag with respect to the solar motion. The data are not consistent with a significant vertical velocity gradient. This points in favor of a formation of the Thick disk by a quick heating of the precursor disk. Finally, no significant rotation velocity is found in the Halo.

Key words. Galaxy: structure - Galaxy: kinematics and dynamics - Galaxy: stellar content - stars: luminosity function, mass function

\section{Introduction}

The formation and evolution of galaxies is one of the forefront problems of Astrophysics. Detailed studies of our own Galaxy are the first step to understand this complex process. In spite of the work done in the recent past on the Galaxy formation, still many unanswered questions remain. The diagnostic to distinguish the different models proposed in literature, is hidden in the observational color-magnitude diagram (CMD), luminosity function (LF), kinematics and dynamics of stellar populations.

Recently, the existence of large databases of proper motions and/or radial velocities as 2MASS, RAVE, CFHTLS, GSC-II, allows the study of large samples of stars. Current issues, that could be addressed with those data, are the rotational character of the Thick Disk and Halo; the presence, if any, of a vertical velocity gradient which might be considered as a relic

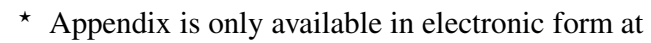
http://www.edpsciences.org of the formation process (Beers et al. 2000; Chiba \& Beers 2001, 2000; Soubiran et al. 2003; Gilmore et al. 2002; Beers \& Sommer-Larsen 1995; Freeman \& Bland-Hawthorn 2002); the existence of a retrograde rotating component in the outer Halo, first suggested by Majewski (1993) and more recently, found in the RR-Lyrae and blue horizontal branch star kinematics (Kinman et al. 2004, 2005). To understand Galaxy formation, it is important to obtain information on the global Galactic kinematics properties (i.e. the velocity ellipsoids), so that deviation from the expected behavior can be assessed.

To address the above issues, a program has been undertaken to analyze space motions and age distributions of stars in the Galaxy. Here we analyze proper motions, magnitudes and colors in the direction of the NGP taken from the GSC-II catalog. For those stars astrometric and photometric data are available.

In Sect. 2 we discuss the data; in Sects. 3 and 4 we present the adopted population synthesis model and kinematic model 
Table 1. Plate material.

\begin{tabular}{rlcccll}
\hline \hline Field & Survey & Center (J2000) & Epoch & Pixel & Color & Emulsion + Filter \\
\hline & & \multicolumn{3}{c}{ NGP $442-l=162.55^{\circ}, b=+86.60^{\circ}$} & \\
XJ442 & POSS-II & $12: 41: 27.0+29: 43: 33$ & 1990.225 & $15 \mu \mathrm{m}$ & $B_{J}$ & IIIaJ + GG385 \\
XP442 & POSS-II & $12: 41: 27.0+29: 43: 33$ & 1990.081 & $15 \mu \mathrm{m}$ & $R_{F}$ & IIIaF + RG610 \\
XI442 & POSS-II & $12: 41: 27.0+29: 43: 33$ & 1997.414 & $15 \mu \mathrm{m}$ & $I_{N}$ & IV-N + RG9 \\
N321 & Quick V & $12: 41: 07.2+29: 12: 15$ & 1983.294 & $25 \mu \mathrm{m}$ & $V_{12}$ & IIaD+Wratten 12 \\
XE321 & POSS-I & $12: 41: 07.2+29: 12: 15$ & 1950.275 & $25 \mu \mathrm{m}$ & $E$ & $103 \mathrm{a}-\mathrm{E}+$ red plexiglass \\
XO321 & POSS-I & $12: 41: 07.2+29: 12: 15$ & 1950.275 & $25 \mu \mathrm{m}$ & $O$ & $103 \mathrm{a}-\mathrm{O}$ unfiltered \\
& & \multicolumn{2}{c}{ NGP 443-l=77.31,$b=+86.11^{\circ}$} & & \\
XJ443 & POSS-II & $13: 04: 23.2+29: 43: 55$ & 1995.234 & $15 \mu \mathrm{m}$ & $B_{J}$ & IIIaJ + GG385 \\
XP443 & POSS-II & $13: 04: 23.2+29: 43: 55$ & 1993.288 & $15 \mu \mathrm{m}$ & $R_{F}$ & IIIaF + RG610 \\
XI443 & POSS-II & $13: 04: 23.2+29: 43: 55$ & 1991.299 & $15 \mu \mathrm{m}$ & $I_{N}$ & IV-N + RG9 \\
N322 & Quick V & $13: 06: 56.1+29: 13: 24$ & 1982.387 & $25 \mu \mathrm{m}$ & $V_{12}$ & IIaD+Wratten 12 \\
XE322 & POSS-I & $13: 06: 56.1+29: 13: 24$ & 1955.288 & $25 \mu \mathrm{m}$ & $E$ & $103 a-E+$ red plexiglass \\
XO322 & POSS-I & $13: 06: 56.1+29: 13: 24$ & 1955.288 & $25 \mu \mathrm{m}$ & $O$ & $103 a-O$ unfiltered \\
\hline
\end{tabular}

respectively; in Sect. 5 we discuss the results, and finally, in Sect. 6 we draw conclusive remarks.

\section{The data}

\subsection{The observational $C M D$}

Our material consists of digitized Schmidt plates from the Northern photographic surveys (POSS-I, Quick-V and POSS-II) carried out at the Palomar Observatory and which have been utilized for the construction of the GSC-II (see Table 1$)^{1}$.

We analyzed two fields in the direction of the NGP. The first, namely $422 \mathrm{c}$, is centered at $(l, b)=\left(162.55^{\circ},+86.60^{\circ}\right)$ and covers an area of $7.1 \mathrm{deg}^{2}$, while the second, $443 \mathrm{c}$, encompasses $19.6 \mathrm{deg}^{2}$ at $(l, b)=\left(77.28^{\circ}, 86.11^{\circ}\right)$. Images were digitized at the STScI (Space Telescope Science Institute) utilizing modified PDS type scanning machines and were processed with the GSC-II software pipeline which provides positions, magnitudes and classification for all the detected objects down to the magnitude limit of the plates.

The absolute position errors are $0 .{ }^{\prime} 2-0 . ' 3$, while relative astrometry attains a precision of 0.1 . Photometric calibrations of the individual plates are based on $B V R_{\mathrm{c}}$ sequences provided by the GSPC-I and GSPC-II (Bucciarelli et al. 2001). The accuracy of the photographic magnitudes is about 0.1-0.2 mag, including systematic errors that can affect objects close to the magnitude limit of the plate or located far from the photometric sequences.

In order to compare the observed GSC-II starcounts with those predicted by the Galaxy model, photographic $B_{J}$, $R_{F}$ magnitudes were transformed to the standard photometry $V, B-V$, by means of color transformations derived from

\footnotetext{
1 The catalogs used for this study are available via http://www. to.astro.it/astrometry/Astrometry/ Galaxy.html or can be requested to A. Spagna.
}
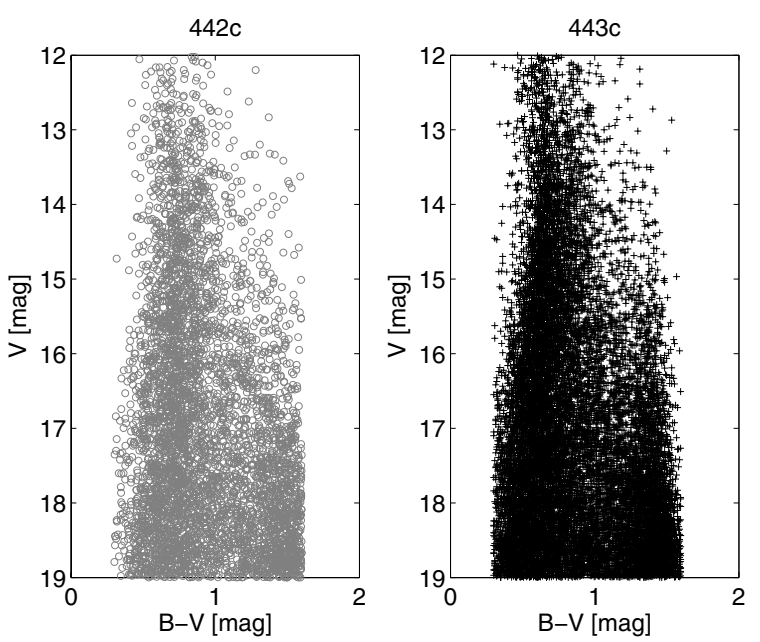

Fig. 1. The observational CMDs for the two fields discussed in the text.

those used for the photometric calibration of the POSS-II plates:

$$
\begin{aligned}
B-V=-0.0372+0.689 \times\left(B_{J}-R_{F}\right) \\
V=B_{J}-0.00299-0.87322(B-V)+0.05116(B-v)^{2} \\
+0.00316(B-V)^{3}
\end{aligned}
$$

Photographic magnitudes, $B_{J}$ and $R_{F}$, and absolute proper motions are available for 14906 stars down to $V=20$ mag in the field $443 \mathrm{c}$ and for 4743 stars in $442 \mathrm{c}$.

The sample is complete for magnitudes brighter than $B_{J} \approx$ 19.5 and $R_{F} \approx 18.5$ corresponding to $V \approx 19.0$, while the completeness abruptly drops below this limit. The observational CMDs are shown in Fig. 1 where only data having both proper motions in $\alpha$ and $\delta$ are shown.

The observed starcounts of both the fields are plotted in Fig. 2. The differences between the luminosity functions of 


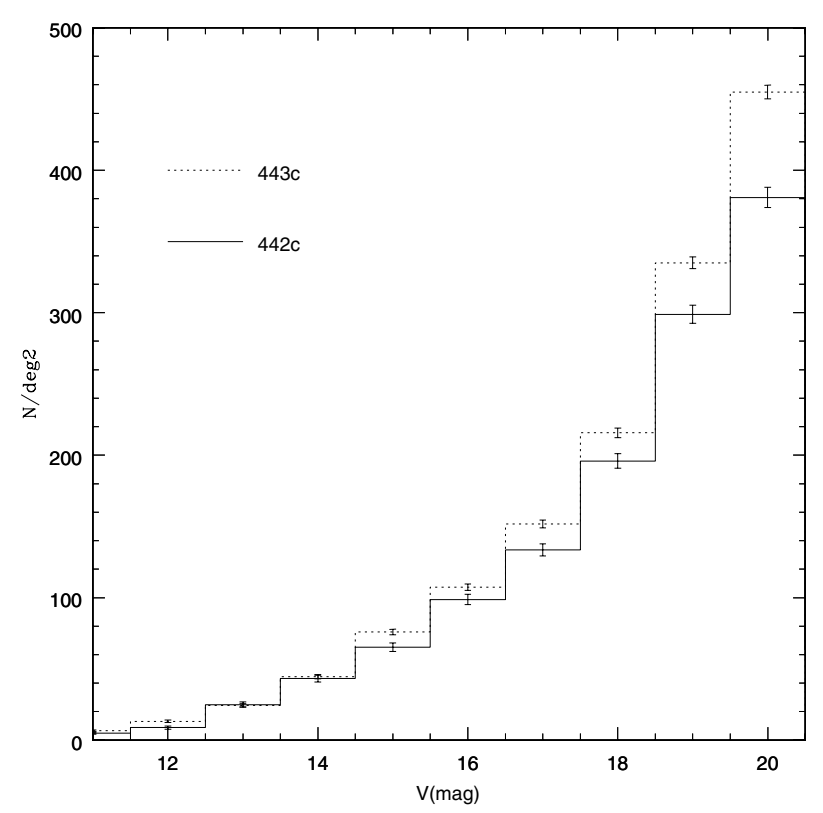

Fig. 2. The luminosity function for the two fields in exam of the GSC-II. The normalization to the area unit is adopted to compare the two distributions.

the two fields might be due to intrinsic inhomogeneity of the Galaxy, but it cannot be excluded that residuals in the photometric corrections are still present. The differences are higher at $V>19$, due to the different completeness of the fields. In Fig. 5 we show the color distributions versus $B-V$ and $B_{J}-R_{F}$ for all the stars brighter than $V=19$. The data have been corrected for a bias $\Delta B_{J} \simeq-0.13$ mag affecting the field $442 \mathrm{c}$ that was revealed because it presented color distributions systematically redder of about $\Delta(B-V) \simeq 0.09$ with respect to those derived from the field $443 \mathrm{c}$ and other regions in the area toward the NGP.

When comparing with the models, this shift is applied to the data in the $V$ magnitude and $(B-V)$ color.

\subsection{The observational proper motions}

Proper motions, $\mu_{\alpha} \cos \delta, \mu_{\delta}$, were computed according to the procedure described by Spagna et al. (1996) using multi-epoch positions measured on POSS-I, Quick-V and POSS-II plates which span an epoch baseline of about 40 years.

The errors on the proper motions are plotted in Figs. 3 and 4 . The bulk of the stars have errors of 3-6 mas/yr, although the mean proper motion error is a function of the magnitude, and it is almost constant, $\sigma_{\mu_{\alpha}} \approx \sigma_{\mu_{\delta}} \approx 2 \mathrm{mas} / \mathrm{yr}$ in the range $12 \lesssim V \lesssim 18$, while increases exponentially at fainter magnitudes (see Fig. 4).

The proper motion space can then be investigated to check the possibility of clustering or bimodal distribution due to systematic errors. Neither evidences of inhomogeneous regions, nor over-densities, nor bimodal distributions are found in the field 442c. Similar conclusion is reached in the case of field $443 \mathrm{c}$. No systematic difference is evident from the proper motion error distribution between the two field 442c and 443c.
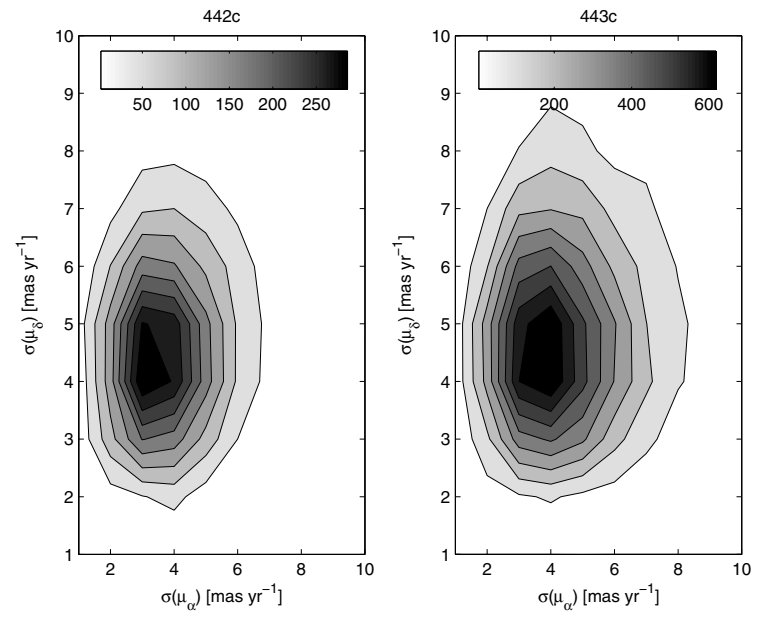

Fig. 3. Contour plot of the proper motion error space. The majority of the stars has errors on the proper motion of $4-5$ mas $r^{-1}$.

\section{Padova Galaxy model}

The Galaxy is modeled with the code already described by Bertelli et al. (1995), where however substantial improvements have been introduced.

The Padova model has been updated including:

1. the usage of new stellar tracks from $Z=0.0001$ till $Z=$ 0.03 with low mass stars down to 0.15 (Girardi et al. 2000).

The $0.1 M_{\odot}$ track is taken from Baraffe et al. (1998);

2. the extinction along the line of sight derived following Drimmel \& Spergel (2001) model obtained from COBE-DIRBE infrared data;

3. the thin disk formed by several populations with different scale height;

4. the velocity space simulated in a consistent way as described in the following sections.

Summarizing, the structure of the Padova Model is as follows. First the synthetic CMDs are generated by Monte Carlo simulations, as described by Vallenari et al. (2000). Then a best fit is produced using a down-hill simplex method in order to derive the scale height and scale length of the populations. A mass density $\left(\rho_{0}\right.$ of Table 4$)$ is then individuated on the basis of the CMD and LF. The Galactic potential $\Phi=\Phi_{\mathrm{TOT}}(R, z)$ is calculated using the mass density to invert the Poisson equation as in Quinn \& Goodman (1986) and Bienayme et al. (1987). We assume a dark matter halo profile as in Helmi \& de Zeeuw (2000), a ISM distribution as in Dehnen \& Binney (1998a), and finally, a bulge mass distribution as in Helmi \& de Zeeuw (2000). The derived potential is used to calculate the radialvertical coupling of the thin disk velocity dispersions $\sigma_{R z}$, the vertical profile of the $\sigma_{z z}$ velocity dispersion and the radial dependence of the azimuthal dispersion $\sigma_{\phi \phi}$. Other radial and vertical dependence are assumed (see Sect. 4.3 for details). Finally velocities and proper motions are generated from the potential. The quality of the fit is evaluated, comparing observations with model predictions by means of a $\chi^{2}$ test. The process is iteratively repeated until a good solution is obtained. More detail can be found in the following sections and in the Appendix. 

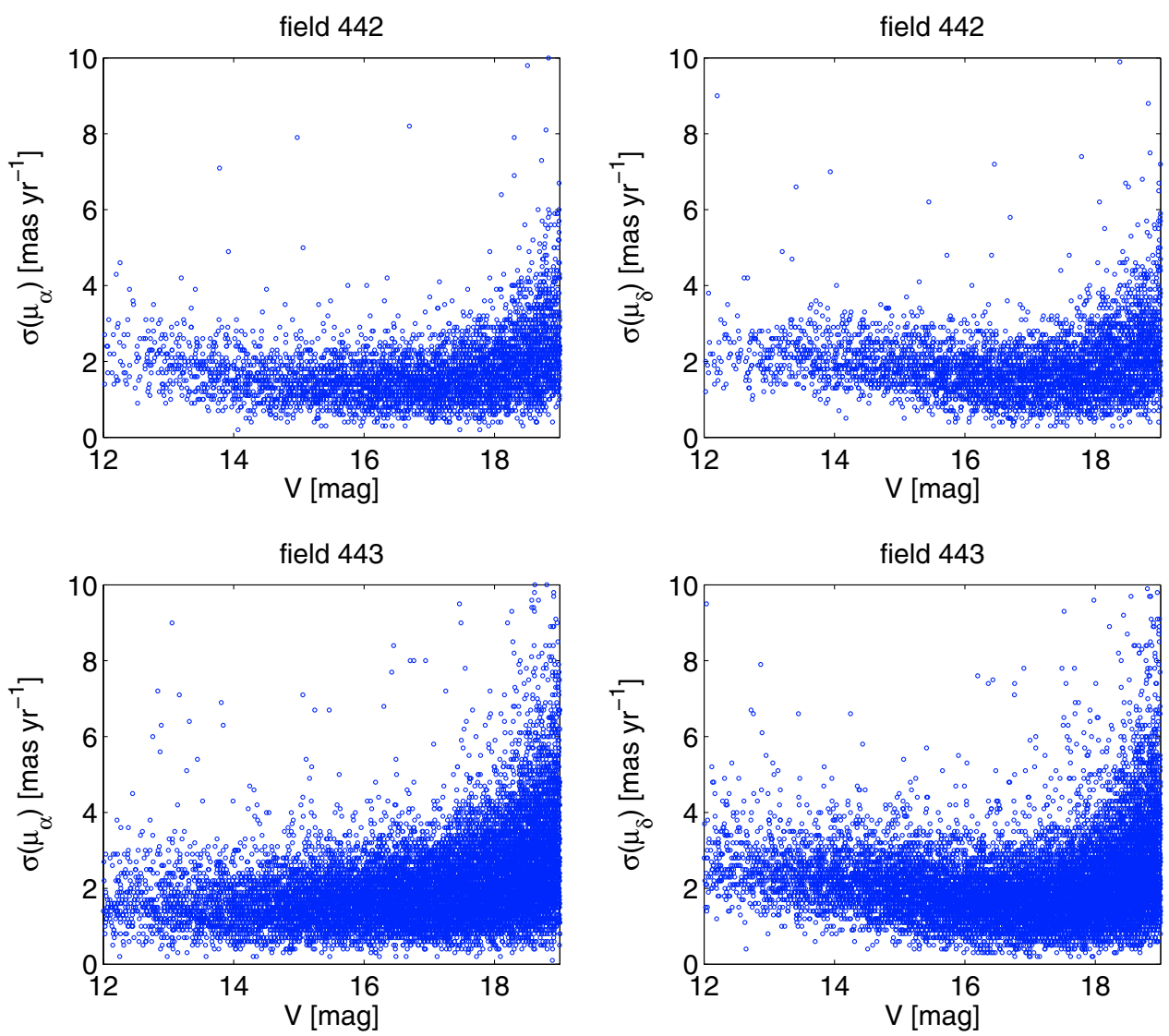

Fig. 4. Proper motion errors as a function of the magnitude for both the components $\mu_{\alpha}$ and $\mu_{\delta}$ and for both the fields $442 \mathrm{c}$ and $443 \mathrm{c}$.

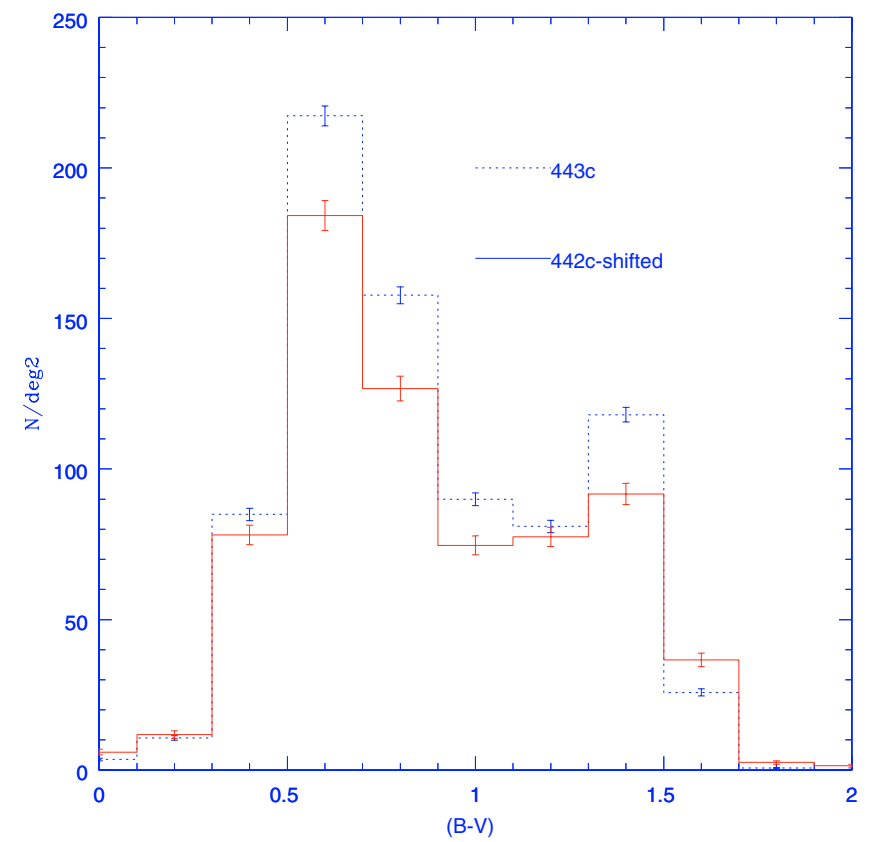

(a)

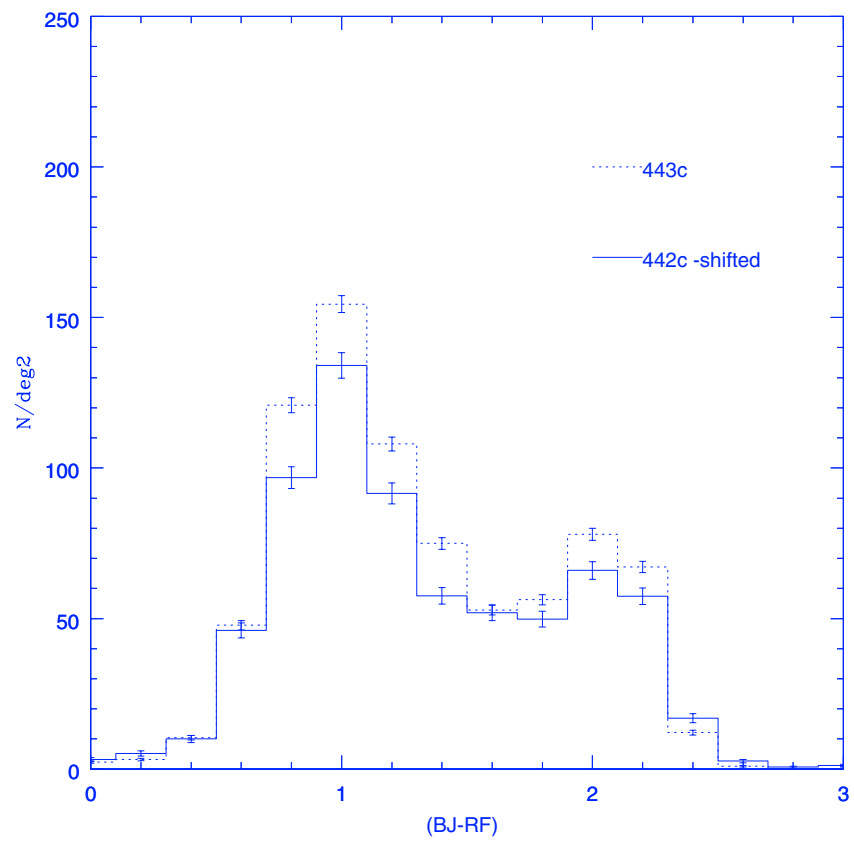

(b)

Fig. 5. Color distributions for the two fields under exam. Panel a) shows the data of both fields in $(B-V)$; $\mathbf{b})$ is the analogous of a) in $\left(B_{J}-R_{F}\right)$. We point out that a shift of $\Delta B_{J}=-0.13 \mathrm{mag}$ is applied to the data of the field $442 \mathrm{c}$ (see text for details). 


\section{Kinematic model}

\subsection{Basic consideration}

The number $N_{k}$ of stars per apparent magnitude, and apparent color unit for Galactic component $k$ at position $\boldsymbol{x}$ and velocity $\boldsymbol{v}$ is given by the fundamental equation of the stellar statistics (von Seeliger 1898; Trumpler \& Weaver 1953), generalized to include the kinematics:

$\frac{\mathrm{d} N_{k}}{\mathrm{~d}^{3} \boldsymbol{v} \mathrm{d}^{3} \boldsymbol{x}}=N_{k} f_{k}(\boldsymbol{x}, \boldsymbol{v})$

where $f_{k}(\boldsymbol{x}, \boldsymbol{v})$ is the Distribution Function (DF) for a population $k$. In the following we consider the reference system in polar cylindrical coordinates $(R, \phi, z)$ in the space of the configurations $(S)$ with the origin of the coordinates in the ideal center of mass of the galaxy. The stationary DF in the phase-space can be expressed as $f_{k}=f_{k}\left(R, z, v_{R}, v_{\phi}, v_{z}\right)$.

This formulation is very similar to the classical one by Schwarzschild (1907). The Schwarzschild DF was initially derived to describe the kinematics of the solar neighborhood. However its use has soon been extended to the whole disk kinematics (Shu 1969; King 1990; Ratnatunga et al. 1989; Mendez \& van Altena 1996). Here we assume as DF a simple trivariate Gaussian having in general cross terms:

$f_{k}(\boldsymbol{x}, \boldsymbol{v})=f_{0, k}(R, z) \exp \left(-\frac{1}{2}(\boldsymbol{a}-\overline{\boldsymbol{a}})^{\mathrm{T}} \Sigma^{(k)}(\boldsymbol{a}-\overline{\boldsymbol{a}})\right)$

where $\boldsymbol{a}=\left(v_{R}, v_{\phi}, v_{Z}\right)$ is a vector in the velocity space, $\overline{\boldsymbol{a}}=$ $\left(0, \bar{v}_{\phi}, 0\right)$ is the mean velocity and $\Sigma_{i j}$ the symmetric dispersion matrix, whose elements are functions of the space coordinates in the meridional plane for the $k$ th-population. While in the Schwarzschild original formulation the velocity dispersion does not depend on the Galactocentric radius (Fricke 1952), in the hydrodynamic approach the DF depends both on the velocity dispersions and positions. From the observational point of view, $\mathrm{S}$ is the natural reference frame of the Galaxy. However it is not convenient to express the DF in this system due to the complexity of the formulation. An easier formulation can be obtained in a system S2 aligned with the main axes of the velocity ellipsoid where the dispersion tensor is diagonal. Once a Gaussian DF is calculated in S2, then a simple change of reference system to $\mathrm{S}$ will conserve the character of a trivariate Gaussian with cross terms. Finally, we will make use of another reference system $(\mathrm{U}, \mathrm{V}, \mathrm{W})$ centered on the Local Standard of Rest, where $\mathrm{U}$ is toward the Galactic center, $\mathrm{V}$ is in the direction of the Galactic rotation, and $\mathrm{W}$ is pointing toward the NGP.

In order to simulate the kinematics of the Galactic components, at any location $P(R, Z)$ the velocity ellipsoid is derived in the reference system $S 2$, whose motion relative to $S$ is expressed by the mean circular speed $\overline{\boldsymbol{V}}_{C, k}=\overline{\boldsymbol{V}}_{C, k}(R, Z)$. Hence a simple transformation matrix in the space of velocities will transform the vectors generated by the DF in S2 to S and finally to the observer system (U, V, W) (see following sections).

\subsection{Geometry of the projected velocities space}

$V_{\mathrm{p}}$, the peculiar velocity vector of a star in the system $\mathrm{S} 2$ centered on $P(R, Z)$ can be expressed as:

$\boldsymbol{V}_{\mathrm{p}}=\overline{\boldsymbol{V}}_{C}\left(R_{\odot}, Z_{\odot}\right)+\boldsymbol{V}_{\odot}+\boldsymbol{V}_{\mathrm{hel}}-\overline{\boldsymbol{V}}_{C, k}(R, Z)$

where $\overline{\boldsymbol{V}}_{C, k}(R, Z)$ is the mean rotational velocity of a star located in $P(R, Z)$ for the population $k, V_{\odot}$ is the solar peculiar velocity vector relative to the LSR, $V_{\text {hel }}$ is the heliocentric velocity vector. The rotation velocity at the Sun in Eq. (4) is:

$\overline{\boldsymbol{V}}_{C}\left(R_{\odot}, Z_{\odot}\right)=\left(\begin{array}{c}0 \\ \boldsymbol{V}_{\mathrm{LSR}} \\ 0\end{array}\right)=\left(\begin{array}{c}0 \\ \left|\overline{\boldsymbol{V}}_{C}\left(R_{\odot}, Z_{\odot}\right)\right| \\ 0\end{array}\right)$.

A velocity vector $V^{\prime \prime}{ }_{p}$ in $\mathrm{S} 2$ is transformed in the system $(\mathrm{U}, \mathrm{V}, \mathrm{W})$ by means of the rotation:

$\boldsymbol{V}_{\mathrm{p}}^{\prime \prime}=\mathbf{E} \boldsymbol{V}_{\mathrm{p}}$

or

$\boldsymbol{V}_{\mathrm{p}}=\mathbf{E}^{-1} \boldsymbol{V}_{\mathrm{p}}^{\prime \prime}=\mathbf{E}^{\mathrm{T}} \boldsymbol{V}_{\mathrm{p}}^{\prime \prime}$

The transformation from $\mathrm{S} 2$ to $(\mathrm{U}, \mathrm{V}, \mathrm{W})$ can be expressed as a function of the angles $\alpha, \beta, \psi$ where $\alpha$ is the angle between the direction of $P(R, Z)$ on the Galactic plane (GP) and the Galactic center (GC), $\beta$ is related to the tilt of the velocity ellipsoid out of the plane (see Eq. (26)), and finally $\psi$ accounts for the vertex deviation. The transformation matrix $\mathbf{E}$ is:

$\mathbf{E}=\mathbf{B C D}$

where

$\mathbf{B}=\left(\begin{array}{ccc}\cos \psi & \sin \psi & 0 \\ -\sin \psi & \cos \psi & 0 \\ 0 & 0 & 1\end{array}\right)$

$\mathbf{C}=\left(\begin{array}{ccc}\cos \beta & 0 & \sin \beta \\ 0 & 1 & 0 \\ -\sin \beta & 0 & \cos \beta\end{array}\right)$

$\mathbf{D}=\left(\begin{array}{ccc}\cos \alpha & \sin \alpha & 0 \\ -\sin \alpha & \cos \alpha & 0 \\ 0 & 0 & 1\end{array}\right)$

Since no vertex deviation is included in the model (see following sections), B is the identity. The Eq. (8) then becomes:

$\mathbf{E}^{\mathrm{T}}=\left(\begin{array}{ccc}\cos \alpha \cos \beta & \sin \alpha & \cos \alpha \sin \beta \\ -\sin \alpha \cos \beta & \cos \alpha & -\sin \alpha \sin \beta \\ -\sin \beta & 0 & +\cos \beta\end{array}\right)$

where the angle $\alpha$ is given by

$\left\{\begin{array}{l}\sin (\alpha)=\frac{r \cos (b) \sin (l)}{R} \\ \cos (\alpha)=\frac{R_{\odot}-r \cos (b) \cos (l)}{R} .\end{array}\right.$ 
Table 2. Adopted values of $\frac{\mathrm{d} \sigma_{i i}}{\mathrm{~d} Z}$ in Eq. (14). The parameters in Eq. (14) are indicated in the first two columns. For each fixed radius the vertical gradient of the two velocity dispersion tensors is indicated in the fourth and fifth columns. The third column gives the vertical ranges.

\begin{tabular}{ccccc}
\hline \hline$\gamma-1$ & $Z_{[\gamma-1]}$ & $Z$ range & $\nabla_{z} \sigma_{R R}$ & $\nabla_{z} \sigma_{\phi \phi}$ \\
\hline 0 & $Z_{0}=0.0$ & $|Z|=0$ & 0 & 0 \\
1 & $Z_{1}=0.0$ & $|Z| \in] 0.0 ; 0.5]$ & 27.2 & 17.4 \\
2 & $Z_{2}=0.5$ & $|Z| \in] 0.5 ; 1.0]$ & 9.78 & 5.44 \\
3 & & $|Z| \geq 1.0$ & 0 & 0 \\
\hline
\end{tabular}

\subsection{The velocity dispersion tensor}

\subsubsection{The thin disk}

No vertex deviation is included in the model, although the importance of the streaming motions on the velocity ellipsoid in the solar neighborhood has been widely evidenced by several authors (Dehnen \& Binney 1998b; Binney \& Merrifield 1998; Delhaye 1965). However the mechanisms responsible for the presence of substructures in the velocity space in the solar vicinity are still under discussion (see e.g. Famaey et al. 2005), as well as the value of the vertex deviation, going from $15^{\circ}$ to $30^{\circ}$ (Dehnen \& Binney 1998b) and its dependence on the age of the disk populations (Famaey et al. 2005; Soubiran et al. 2003).

Then the azimuthal and radial velocities will be considered un-coupled or equivalently $\sigma_{R \phi}^{2}=\overline{v_{R}\left(v_{\phi}-\bar{v}_{\phi}\right)} \equiv 0$.

It can be shown that in a cool disk the azimuthal and vertical velocities are approximately un-coupled, or equivalently that the short axis of the velocity ellipsoid remains aligned with a cylindrical coordinate system so that $\sigma_{\phi Z}^{2}=\overline{\left(v_{\phi}-\bar{v}_{\phi}\right) v_{Z}} \sim 0$. This is strictly true because of the symmetry on the galactic plane (Aoki 1965, 1985; Hunter 1975, 1979; Vandervoort 1975).

Following Fuchs \& Wielen (1987) the vertical profile of the velocities dispersion $\sigma_{i j}$ can be approximated by a recurrence relation on the index $\gamma$ as:

$\sigma_{i i}^{(\gamma)}(R, z)=\nabla_{z} \sigma_{i i}^{(\gamma)}(R, z) \cdot\left(|z|-z^{(\gamma-1)}\right) \ldots$

$\ldots+\sigma_{i i}^{(\gamma-1)}\left(R, z^{(\gamma-1)}\right)$

for $\gamma=1,2,3$ and where $i i=R, \phi$. The adopted coefficients $\nabla_{z} \sigma_{i i}^{(\gamma)}(R, z)$ as function of $\gamma$ are taken as in Fuchs \& Wielen (1987) and are given in Table 2.

For all reasonable gravitational potentials, $\sigma_{R R}^{2}>\sigma_{z z}^{2}$. It can be proved that $\sigma_{z z}^{2}$ is minimum on the plane, i.e. $\partial \sigma_{z z}^{2} / \partial z=0$ (Hill et al. 1979; Wainscoat et al. 1989; Fuchs \& Wielen 1987; van der Kruit 1988). In the solar neighborhood $\left(R=R_{\odot}\right)$, at small $z$ a Taylor expansion in $\sigma_{z z}^{2}$ in the vertical direction gives:

$\sigma_{z z}^{2}\left(R_{\odot}, z\right) \simeq \sigma_{z z}^{2}\left(R_{\odot}, 0\right)+\frac{1}{2} z^{2} \frac{\partial^{2} \sigma_{z z}^{2}\left(R_{\odot}\right)}{\partial z^{2}}+O\left(z^{3}\right)$

where $O\left(z^{3}\right)$ represents higher order derivatives. It follows:

$\frac{\sigma_{z z}^{2}\left(R_{\odot}, z\right)}{\sigma_{z z}^{2}\left(R_{\odot}, 0\right)} \simeq 1+\frac{\lambda\left(R_{\odot}\right)}{2 R_{\odot}}(\alpha-1)\left\|\left.\frac{\partial \ln \sigma_{z z}^{2}}{\partial R}\right|_{\left(R_{\odot}, 0\right)}\right\| z^{2}$ where $\lambda$ is derived from the Galactic potential (see Eq. (22)) as in Amendt \& Cuddeford (1991).

Supposing that $\sigma_{z z}^{2} \propto \rho$ in the plane, as in van der Kruit $\&$ Searle (1982), and that $\rho$ follows an exponential law with constant scale length $h_{R}$ we have

$\frac{\sigma_{z z}^{2}\left(R_{\odot}, z\right)}{\sigma_{z z}^{2}\left(R_{\odot}, 0\right)} \simeq 1+\frac{\lambda\left(R_{\odot}\right)}{2 R_{\odot}}\left(\frac{\sigma_{R R}^{2}\left(R_{\odot}, 0\right)}{\sigma_{z z}^{2}\left(R_{\odot}, 0\right)}-1\right) \frac{z^{2}}{h_{R}}$

which describes the non isothermal case as

$$
\begin{aligned}
& \sigma_{z z}^{2}\left(R_{\odot}, z\right) \simeq \sigma_{z z}^{2}\left(R_{\odot}, 0\right) \\
& \quad+\left(\frac{\lambda\left(R_{\odot}\right)}{2 R_{\odot}}\left(\frac{\sigma_{R R}^{2}\left(R_{\odot}, 0\right)}{\sigma_{z z}^{2}\left(R_{\odot}, 0\right)}-1\right) \frac{z^{2}}{h_{R}}\right) \sigma_{z z}^{2}\left(R_{\odot}, 0\right) .
\end{aligned}
$$

This formulation takes into account the non-isothermal structure of the Thin Disk. Generalizing the kinematical characteristic of the solar neighborhood to the whole disk of the Galaxy, we can write Eq. (15) as:

$\sigma_{z z}^{2}(R, z) \simeq \sigma_{z z}^{2}(R, 0)+\left.\frac{\lambda(R, \Phi)\left(\sigma_{R R}^{2}-\sigma_{z z}^{2}\right)}{2 \cdot h_{R} \cdot R}\right|_{(R, 0)} z^{2}$

where $\Phi$ is the Galactic Potential (see Sect. 4.3 .3 where the dependence of $\lambda$ from the potential will be discussed). The adopted approximation of the vertical profile is in agreement with the Bahcall (1984a) data up to $1 \mathrm{kpc}$ from the plane.

The radial profile terms in the plane are described as in Lewis \& Freeman (1989). They assume an exponential radial dispersion profile corresponding to an anisotropic model. Under the hypothesis that $\sigma_{R R}^{2[\gamma-1=0]} / \sigma_{z z}^{2[\gamma-1=0]}=$ const. they obtain:

$\sigma_{R R}^{2[\gamma-1=0]}(R, 0)=\sigma_{R R, \odot}^{2} \exp \left(\frac{-\left(R-R_{\odot}\right)}{h_{R}}\right)$
$\sigma_{z z}^{2[\gamma-1=0]}(R, 0)=\sigma_{z z, \odot}^{2} \exp \left(\frac{-\left(R-R_{\odot}\right)}{h_{R}}\right)$.

The azimuthal dispersion profile depends on the rotation curve as in Binney \& Merrifield (1998):

$\sigma_{\phi \phi}^{2[\gamma-1=0]}(R, 0)=\frac{1}{2}\left(1+\frac{\mathrm{d} \ln V_{\mathrm{C}}(R)}{\mathrm{d} R}\right) \sigma_{R R}^{2[\gamma=0]}(R, 0)$.

Finally, the only off-diagonal term we take into account is related to the vertical tilt, i.e. the coupling of the velocities in $R-z$ and will be discussed in the following sections.

\subsubsection{Age-velocity relation}

As it's well known an age-velocity dispersion relation seems to exist for disk stars. A series of spiral transient events can strongly mix stars in the radial direction (Sellwood \& Binney 2002). In addition to radial heating stars experience a vertical disk heating. Their vertical velocity dispersion increases as they age. This is believed to occur through a combination of in-plane spiral arm heating and scattering off giant molecular clouds (Carlberg \& Sellwood 1985). Then the vertical structure is related to the age distribution of the population (Gomez et al. 1997). When the amplitude of the random motions increases, the stars become less vulnerable to heating by transient 
Table 3. The local values of the velocity ellipsoid taken from Rocha-Pinto et al. (2004).

\begin{tabular}{cccc}
\hline \hline Age [Gyr] & $\sigma_{R R, \odot}$ & $\sigma_{\phi \phi, \odot}$ & $\sigma_{z z, \odot}$ \\
\hline $0-1$ & 25 & 16 & 9 \\
$1-3$ & 37 & 22 & 17 \\
$3-5$ & 45 & 23 & 19 \\
$5-7$ & 51 & 30 & 22 \\
$7-10$ & 52 & 33 & 27 \\
\hline
\end{tabular}

spiral waves, and the heating process is expected to saturate. Inside this general framework, however, no complete agreement is found among authors concerning the details of the process (Wielen 1974a,b; Mayor 1974; Carlberg et al. 1985; Knude et al. 1987; Stromgren 1987; Meusinger et al. 1991; Wielen et al. 1992; Dehnen \& Binney 1998b; Fuchs et al. 2001; Gómez et al. 1998; Rocha-Pinto et al. 2004; Nordström et al. 2004). On the observational ground, it is matter of debate whether the effect saturates or not after 3-4 Gyr (Gomez et al. 1997; Nordström et al. 2004; Rocha-Pinto et al. 2004). Table 3 presents the age-velocity relation derived by Rocha-Pinto et al. (2004) that will be used in the following as starting guess for the down-hill simplex fit.

\subsubsection{Thin disk vertical tilt}

The thin disk tilt term is described following Amendt \& Cuddeford (1991) who analytically link the vertical-coupling term of the velocities ellipsoid to the potential, and to the density distribution:

$\sigma_{R z}^{2}(R, z) \cong \sigma_{R z}^{2}\left(R_{\odot}, 0\right)+z \frac{\partial \sigma_{R z}^{2}\left(R_{\odot}, 0\right)}{\partial z}+o\left(z^{2}\right)$.

The first term of the sum is null by symmetry when $z=0$ and the last term is given by:

$\frac{\partial \sigma_{R z}^{2}(R, 0)}{\partial z}=\lambda(R)\left(\frac{\sigma_{R R}^{2}-\sigma_{z z}^{2}}{R}\right)(R, 0)$

where

$\lambda(R)=\left(\frac{R^{2} \Phi_{, R z z}}{3 \Phi_{, R}+R \Phi_{, R R}-4 R \Phi_{, z z}}\right)(R, z=0)$

where $\Phi=\Phi_{\text {TOT }}(R, z)$ is the total potential of the Galaxy (see Appendix) and where $\Phi_{, R z z}, \Phi_{, R}, \Phi_{, R R}, \Phi_{, z z}$ are the derivatives of the potential. $\lambda$ is 1 in the case of a spherical potential, when the velocity ellipsoid is pointing toward the Galactic center, while $\lambda$ is 0 for a cylindrical potential when the velocity ellipsoid is always parallel to the Galactic plane. Amendt \& Cuddeford (1991) show that $\lambda$ is related to the mass gradient in the Galactic plane. A galaxy with a flat rotation curve and a flat mass distribution has $\lambda(R)=0.5$ at $z=0$.

Out of the plane of symmetry of the galaxy the vertical tilt of the velocity ellipsoid results in a rotation of an angle $\beta$ around $v_{\phi}$ in S2 (defined in Sect. 4.1). $\beta$ can be expressed as a function of the velocity dispersions in $\mathrm{S}$. If $\boldsymbol{v}_{\mathrm{p}}^{\prime \prime}=\left(v_{R}^{\prime \prime}, v_{\phi}^{\prime \prime}, v_{z}^{\prime \prime}\right)$ is the vector in $\mathrm{S} 2$, then the corresponding vector in $\mathrm{S}$ is $\boldsymbol{v}_{\mathrm{p}}=\left(v_{R}, v_{\phi}, v_{z}\right)=\mathbf{C} \boldsymbol{v}^{\prime \prime}{ }_{\mathrm{p}}$ where the rotation matrix $\mathbf{C}$ is defined in Sect. 4.2. If $\left(\sigma_{i i}\right)^{2}=\overline{\left(v_{i i}-\bar{v}_{i i}\right)^{2}}$ we find that in S2 the DF of the disc components can be expressed by Eq. (3) where the dispersion tensor is of the form:

$\Sigma_{i j}=-\frac{1}{2} \cdot\left(\begin{array}{ccc}\left(\sigma_{R R}^{\prime \prime}\right)^{-2} & 0 & 0 \\ 0 & \left(\sigma_{\phi \phi}^{\prime \prime}\right)^{-2} & 0 \\ 0 & 0 & \left(\sigma_{z z}^{\prime \prime}\right)^{-2}\end{array}\right)$.

The normalization factor $f_{k, 0}$ can be expressed as:

$f_{k, 0}(R, z)=\frac{1}{(2 \pi)^{3 / 2} \sigma_{R R}^{\prime \prime}(R, z) \sigma_{\phi \phi}^{\prime \prime}(R, z) \sigma_{z z}^{\prime \prime}(R, z)}$.

The simplest way to derive the components of the velocity dispersion tensor is to find its eigenvalues: solving the characteristic equation for the velocity dispersion tensor $\operatorname{det}(\boldsymbol{\Sigma}-\lambda \boldsymbol{I})=0$, one finds

$\lambda_{1}=\frac{1}{2}\left(\sigma_{R R}^{2}+\sigma_{z z}^{2}-\sqrt{\left(2 \sigma_{R z}^{2}\right)^{2}+\left(\sigma_{R R}^{2}-\sigma_{z z}^{2}\right)^{2}}\right) \equiv\left(\sigma_{R R}^{\prime \prime}\right)^{-2}$
$\lambda_{3}=\frac{1}{2}\left(\sigma_{R R}^{2}+\sigma_{z z}^{2}+\sqrt{\left(2 \sigma_{R z}^{2}\right)^{2}+\left(\sigma_{R R}^{2}-\sigma_{z z}^{2}\right)^{2}}\right) \equiv\left(\sigma_{z z}^{\prime \prime}\right)^{-2}$

while $\sigma_{\phi \phi}^{2} \equiv\left(\sigma_{\phi \phi}^{\prime \prime}\right)^{2}$. We can write:

$\left(\sigma_{R R}^{\prime \prime}\right)^{2}=\left(\eta^{-}(R, z)\right)^{-1}$

$\left(\sigma_{z z}^{\prime \prime}\right)^{2}=\left(\eta^{+}(R, z)\right)^{-1}$

where

$\eta^{\mp}(R, z)=\frac{1}{2}\left(\sigma_{R R}^{2}+\sigma_{z z}^{2} \mp \sqrt{\left(2 \sigma_{R z}^{2}\right)^{2}+\left(\sigma_{R R}^{2}-\sigma_{z z}^{2}\right)^{2}}\right)$.

The above equations describe how the velocity dispersions change in S2 as a function of the observable profile in S due to the tilt of the velocity ellipsoid out of the symmetry plane of the MW. The inclination angle $\beta$ can be obtained imposing that in the reference frame $\mathrm{S} 2$ where the DF is a normal distribution with only diagonal dispersion terms, $v_{R}$ and $v_{z}$ are statistically independent, in the sense that $\overline{v_{R} v_{z}}=0 . \beta$ comes out to be:

$2 \beta=\arctan \left(\frac{2 \sigma_{R z}^{2}}{\sigma_{R R}^{2}-\sigma_{z z}^{2}}\right)$

where $\sigma_{R z}^{2}, \sigma_{R R}^{2}$ and $\sigma_{z z}^{2}$ are functions of the position (i.e. Bienaymé 1999).

\subsection{Thick disk DF}

Concerning the Thick Disk, neither age nor position dependence in the velocity dispersion are ascertained. We describe the Thick Disk velocity ellipsoid as an anisotropic but isothermal DF. Little information is available on the thick disk kinematics. In particular it is not clear whether the velocity ellipsoid presents or not a vertical tilt. The data suggest a vertex deviation near to zero (Soubiran et al. 2003). For those reasons, neither vertical tilt nor vertex deviation is included in the 
model. A simple trivariate Gaussian with no cross terms (see e.g. Méndez et al. 2000) is assumed to give a description of the velocity distribution:

$f_{\text {thick }}(\boldsymbol{v}, \boldsymbol{x})=f_{\text {thick }, 0} \exp \left((\boldsymbol{a}-\overline{\boldsymbol{a}})^{\mathrm{T}} \cdot \boldsymbol{\Sigma} \cdot(\boldsymbol{a}-\overline{\boldsymbol{a}})\right)$

where

$\Sigma=\frac{1}{2}\left(\begin{array}{ccc}\sigma_{R R}^{-2} & 0 & 0 \\ 0 & \sigma_{\phi \phi}^{-2} & 0 \\ 0 & 0 & \sigma_{z z}^{-2}\end{array}\right)$

where $f_{\text {thick, } 0}$ is the normalization factor and $\Sigma$ is the thick disk dispersion matrix, whose elements are not functions of the space coordinates but are regarded as constant.

\subsection{Halo DF}

We approximate the Halo with an isothermal anisotropic spheroid. A Gaussian DF is used even if more sophisticated approaches have been presented in literature, i.e. a DF depending on the energy and the angular moment $f=f(E, L)$ such as in the Osipkov-Merrit models (Osipkov 1979; Merritt 1985b,a) or Michie models (Michie 1963). Our simple approach allows us to easily introduce a constant rotation, a skew-rotational velocity or a two-components model for the inner and outer Halo (e.g. Chiba \& Beers 2001). The DF can be written as a trivariate Gaussian with no cross terms in the LSR of any given point in the space:

$f_{\text {halo }}(\boldsymbol{v})=f_{\text {halo }, 0} \exp \left(\boldsymbol{a}^{\mathrm{T}} \cdot \boldsymbol{\Sigma} \cdot \boldsymbol{a}\right)$

where

$\boldsymbol{\Sigma}=\frac{1}{2}\left(\begin{array}{ccc}\sigma_{R R}^{-2} & 0 & 0 \\ 0 & \sigma_{\phi \phi}^{-2} & 0 \\ 0 & 0 & \sigma_{z z}^{-2}\end{array}\right)$

are position independent velocity dispersions for the halo.

\subsection{The circular velocity}

- The thin disk. The module of the mean circular velocity of the thin disk component $k,\left|\overline{\boldsymbol{V}}_{\boldsymbol{C}, k}(R, z)\right|$, at $z=0$ is:

$$
\left|\overline{\boldsymbol{V}}_{\boldsymbol{C}, k}(R, z)\right| \cong\left|\overline{\boldsymbol{V}}_{\boldsymbol{C}, k}(R, 0)\right|=\sqrt{V_{\mathrm{c}}^{2}(R)-\sigma_{\phi \phi, k}^{2}+\ldots}
$$

$$
\ldots+\overline{\left(1+\frac{2 R}{h_{R, \text { thin }}}+\frac{R}{\sigma_{R R, k}^{2}} \frac{\partial \sigma_{R z, k}^{2}}{\partial z}\right) \sigma_{R R, k}^{2}}
$$

where the vertical tilt of the ellipsoid is calculated following Eq. (22).

This formulation which is rigorously valid at $z=0$ can be regarded as a reasonable approximation up to a few hundred parsecs out of the plane.
- The thick disk. We assume:

$\left|\overline{\boldsymbol{V}}_{\text {Cthick }}(R, z)\right| \equiv$ const.

Alternatively the vertical gradient can be calculated from the radial Jeans equation. We derive:

$\left|\overline{\boldsymbol{V}}_{C}(R, z)\right|=\sqrt{\left(1-\frac{R}{h_{R, \text { thick }}}\right) \sigma_{R R}^{2}-\sigma_{\phi \phi}^{2} \cdots}$

$\ldots+R \frac{\partial \Phi_{\mathrm{TOT}}(R, z)}{\partial R}$

where $h_{R \text {,thick }}$ is the thick-disk scale length (see Appendix for details on the potential).

- The halo. We assume for the halo constant circular mean velocity analogously to Eq. (32).

\section{Results}

The comparison of the observational CMD, color distribution and proper motion with the simulations is made minimizing the $\chi^{2}$ distribution in the several magnitude ranges using the down-hill simplex method.

In this section, first we briefly present the down-hill simplex method, then we discuss the results.

\subsection{The down-hill simplex method}

The down-hill simplex method is a direct search method of optimization that works for stochastic problems. It is based on evaluating a function at the vertices of a simplex, then iteratively shrinking the simplex as better points are found until some desired bound is obtained. Since its publication by Nelder \& Mead (1965), the simplex algorithm has become one of the most widely used methods. We use this method minimizing the $\chi^{2}$ function in a parameter-space having $N$ dimensions. In the following, the observational CMD is divided into magnitude and color bins of size $\Delta(B-V)=0.2 \mathrm{mag}$ and $\Delta V=1.0 \mathrm{mag}$, where the fitting parameters are the scale heights of the Thin and Thick Disk and the relative percentages of the populations. Concerning the kinematics, the $\sigma_{i i, \odot}$ are regarded as free parameters and then derived using the downhill simplex method fitting the observational distributions of proper motions $\mu_{\alpha}$ and $\mu_{\delta}$ in magnitude bins. We point out that in the simulations, observational errors on magnitudes, colors, and proper motions as derived from the data (see Sect. 2) are taken into account. To prevent settling on local rather than global minima, the simplex is first started from a random position in the parameter space for the thick disk and halo, while we make use as first guess of the local values of the thin disk velocity ellipsoids of the populations defined in Table 3. When a possible solution is obtained, the simplex is re-started from a position very close to it. The uncertainties on the parameters are derived using Boot-strap Method simulations, where a fraction equals to $1 / e$ of the original data are randomly substituted within the errors and the new data sample is re-analyzed (Efron 1982). 
Table 4. Column 1 indicates the number of the Model, Cols. 2 and 3 present the IMF slope used in the simulation for the Thin and Thick disk respectively in the mass range $0.2-m_{\mathrm{c}}$. Column 4 indicates the mass $m_{\mathrm{c}}$ at which the IMF slope of the Thin disk changes. Column 5 gives the scale height for the Thick Disk. Columns 6 and 7 indicate the used fraction of binaries stars. The last column gives the chi-square merit function.

\begin{tabular}{cccccccc}
\hline \hline Models & IMF $\alpha$ & IMF $\alpha$ & $m_{\mathrm{c}}$ & $h_{z}$ & \multicolumn{2}{c}{ Binaries } & $\chi^{2} / N$ \\
& Thin disk & Thick disk & $M_{\odot}$ & Thick disk [kpc] & Thin disk & Thick disk & \\
\hline$(1)$ & Kroupa & Kroupa & 0.5 & 0.9 & No & No & 6.2 \\
$(2)$ & Kroupa & -0.5 & 0.5 & 0.7 & No & No & 2.7 \\
$(3)$ & 1.1 & 1.1 & 0.8 & 0.8 & No & No & 3.0 \\
$(4)$ & 0.9 & 0.5 & 0.6 & 1.2 & No & No & 7.8 \\
$(5)$ & 0.9 & 0.5 & 0.7 & 1.5 & No & No & 7.1 \\
$(6)$ & 0.9 & 0.5 & 0.8 & 1.5 & No & No & 7.0 \\
$(7)$ & Kroupa & 0.5 & 0.5 & 0.9 & $40 \%$ & $40 \%$ & 5.2 \\
$(8)$ & 0.9 & 0.5 & 0.8 & 0.9 & $40 \%$ & $40 \%$ & 2.2 \\
$(9)$ & 0.9 & 0.5 & 0.6 & 0.9 & No & $40 \%$ & 3.4 \\
$(10)$ & 0.9 & 0.5 & 0.7 & 0.9 & No & $40 \%$ & 3.1 \\
$(11)$ & 0.9 & 0.5 & 0.8 & 0.7 & No & $40 \%$ & 2.9 \\
\hline
\end{tabular}

\subsection{CMD and LF analysis}

In the following, a detailed analysis of the two fields is presented.

Table 4 shows the most interesting solutions obtained using the standard Kroupa (2001b,a) IMF for all the populations.

The best solution with a Kroupa IMF and when binary stars are not taken into account is obtained for a Thick disk scale height $h_{z \text {,thick }}=0.9 \pm 0.2 \mathrm{kpc}$ (see model 1 of Table 4$)$.

Figure 6 shows the poor quality of the fit, comparing the observational color distribution with the results of model (1). The data show a bimodal distribution in the magnitude range $V \in$ [17-19] mag, with the stars distributed in two regions, one redder than $(B-V) \sim 1.2$ and one bluer than $(B-V) \sim 0.8$. While the blue peak is reproduced, the red one is poorly fitted. Additionally, when the number of faint stars is reproduced, then in the magnitude ranges $V \in[13-15]$ mag and $V \in$ [15-17] mag the number of stars in the central peak is greatly underestimated. Looking at the simulated CMD (see Fig. 7) it is clear that, due to their age, age distribution, metallicity and spatial distribution, Halo, Thin and Thick Disk are mainly located in different regions of the CMD, although it is not really possible to separate them. Stars brighter than $V=15$ are mainly Thin Disk population, objects in the range $V \in$ [15-17] are a mixture of Thin and Thick Disk population, whereas the Halo contribution becomes significant for $V>17$. In order to improve the quality of the fit at brighter magnitudes, one of the most critical parameters is the slope of the IMF. Figure 8 presents the simulated CMD where the mass range is brought into evidence. The relative percentage of bright blue stars to faint red objects can be modified changing the IMF, since the blue and the red part of the CMD are occupied by stars of different mass ranges. Regarding the IMF slope as a free parameter, when no binary stars are included, we find out that better merit functions are obtained when the IMF slope changes at $m_{\mathrm{c}}=0.8 M_{\odot}$ for the Thin and Thick disk instead that at the canonical Kroupa value of $0.5 M_{\odot}$ (see model 3 ). We derive in the lower mass range, $0.2-0.8 M_{\odot}, \alpha=1.1 \pm 0.3$ whereas for masses higher than $0.8 M_{\odot}$ no need is found to change the standard value of $\alpha=2.3$ (model 4 of Table 4 ). In this notation the Salpeter slope is 2.35. We remind that Kroupa (2003) suggests a slope $\alpha=1.1 \pm 0.7$ in the mass range $0.1-0.5 M_{\odot}$ and $\alpha=2.2 \pm 0.3$ for more massive stars. The Thick disk scale height turns out to be $h_{z \text {,thick }}=0.8 \pm 0.1 \mathrm{kpc}$. The importance of the change of $m_{\mathrm{c}}$ is pointed out by the fact that when binaries are not included, and $m_{\mathrm{c}}=0.5 M_{\odot}$, then the best fit Thick disk IMF turns out to be rising with a slope $\alpha=-0.5 \pm 0.2$ from $0.8 M_{\odot}$ and the Thick disk scale height becomes $0.7 \pm 0.2 \mathrm{kpc}($ model 2$)$.

The simulations of the previous paragraph do not take into account the presence of binary stars. Kroupa (2001b) has pointed out the importance of binaries in the IMF determinations. We recall that the predicted multiplicity of stars depends on the spectral type and is varying from $30-40 \%$ for the M-dwarf stars (Fischer \& Marcy 1992) to 59\% for O-stars (Mason et al. 1998) till 65\% of GV-stars (Duquennoy et al. 1991; Duquennoy \& Mayor 1991). Models taking into account a percentage of $40 \%$ of binaries have been tested against star counts. A poor solution is derived when the standard Kroupa IMF is adopted for the Thin Disk at varying parameters of the Thick Disk (IMF slope and scale height) (see for instance model 7). The most convincing solution (model 8) is found including $40 \%$ of binary stars for the Thin Disk IMF slope $\alpha=0.9 \pm 0.3$ in the mass range $0.2-0.8 M_{\odot}$, when the Thick Disk IMF slope is $\alpha=0.5$. This is in agreement with Reylé $\&$ Robin (2002) who derive $\alpha=0.5$ for the thick disk population in the mass range $0.2-0.8 M_{\odot}$. In this case, the Thick Disk scale height is $0.9 \pm 0.1 \mathrm{kpc}$ in agreement with the results obtained by other authors (we quote among others Spagna et al. 2005; Reylé \& Robin 2002; Larsen \& Humphreys 2003; Ng et al. 1997). The merit function $\chi^{2} / N$ is definitely better than in the previous solutions. Figure 9 compares the observational 


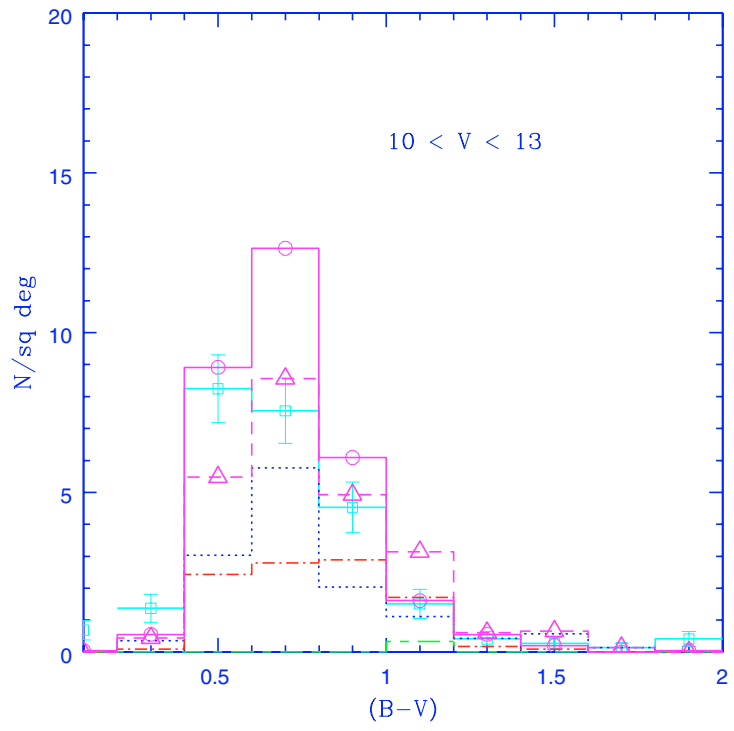

(a)

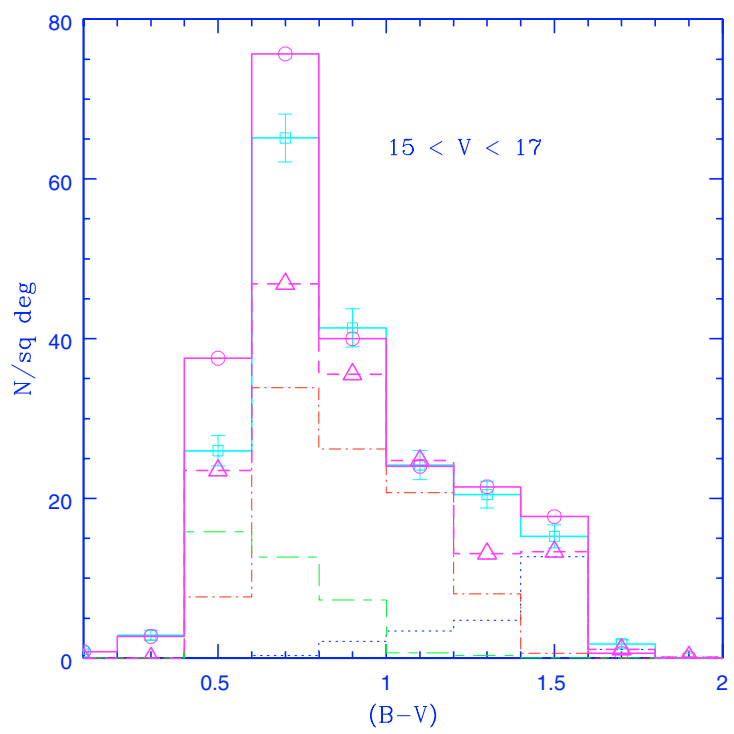

(c)

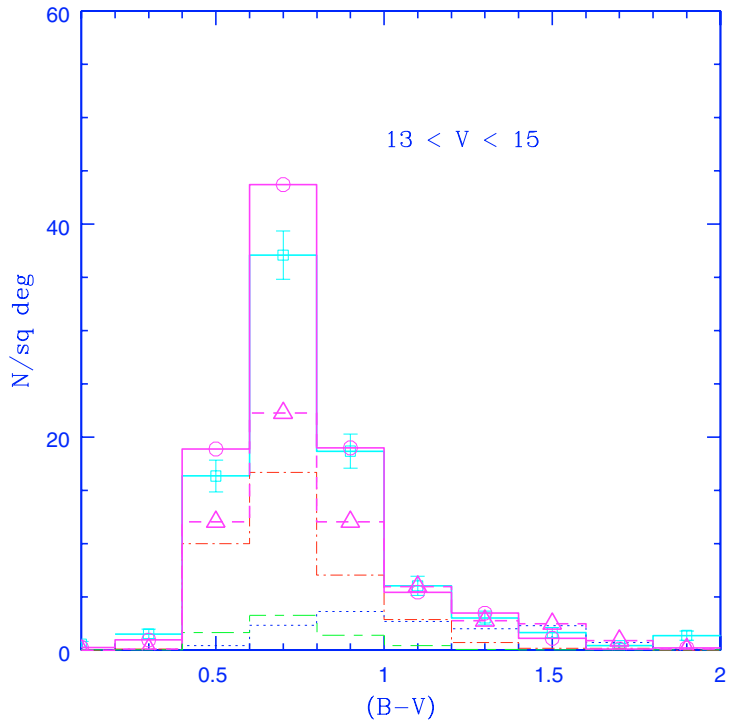

(b)

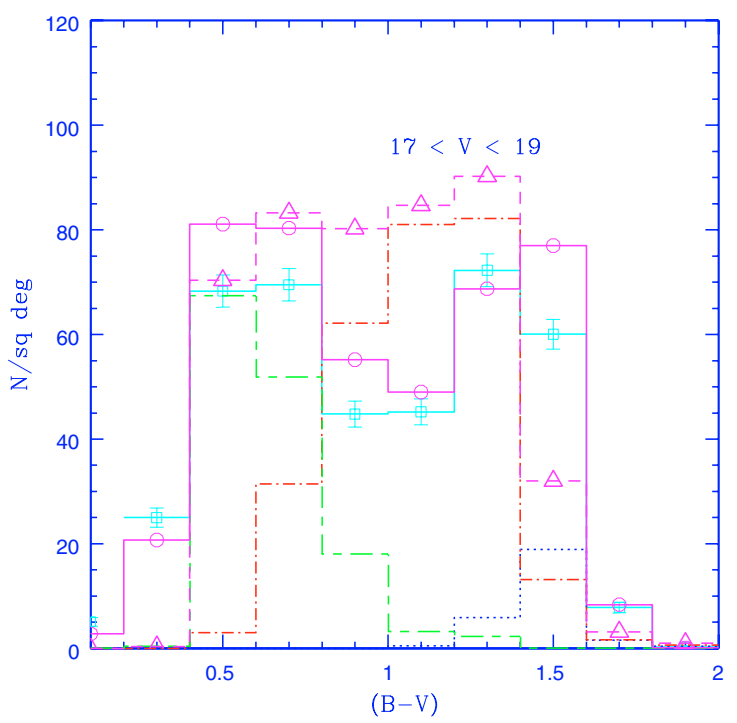

(d)

Fig. 6. Comparison of the observational and simulated color distribution in different magnitude range, for the model (1) of Table 3 . The solid line with error-bars and squares represents the data of field F2, the solid line with circles gives the data of F3, the long-short dashed line indicates the halo stars, the dashed-dotted line shows thick disk stars, the dotted line represents the thin disk population. Finally dashed line with triangles is the total simulated LF (see text for details).

color distribution with the best solution model for magnitudes brighter than $V=19$. The importance of including binary stars is pointed out by the comparison between the models (6), (8) and (11).

The IMF slope at low masses is still under debate. Holtzman et al. (1999) find $\alpha=0.9$ for $0.3-0.7 M_{\odot}$ for the Bulge population. Chabrier (2003) suggests for the solar vicinity a slope $\alpha=1$ for stars in the mass range $0.1-0.35 M_{\odot}$, and $\alpha=2$ for higher masses. Schultheis et al. (2005) derive a change in the IMF slope for $m<0.2-0.25 M_{\odot}$ for the Thin disk single stars. If the stars in the Galactic disk are born in embedded clusters rather than in isolation (Lada \& Lada 2003), then the young cluster IMF can give information about the disk IMF. In most star forming regions and open clusters such as Trapezium, IC 348, Taurus, the IMF rises from high masses down to about $0.8 M_{\odot}$, then rises more slowly down to $0.1-0.2 M_{\odot}$ and finally declines in the sub-stellar regime (Luhman \& Potter 2006, and references therein). Muench et al. (2003) obtain $\alpha=1.2$ in the mass range $0.1-0.7 M_{\odot}$ in two young clusters. Recently, Luhman et al. (2003) and Luhman (2004) derive in Taurus an IMF showing a peak near $0.8 M_{\odot}$ and then steadily declining toward lower masses. This is in agreement with the results from the earlier surveys of Taurus (Briceño et al. 2002) and is usually interpreted in terms of different average Jeans masses between Taurus and other denser clusters resulting in the shift in peak mass as proposed by 


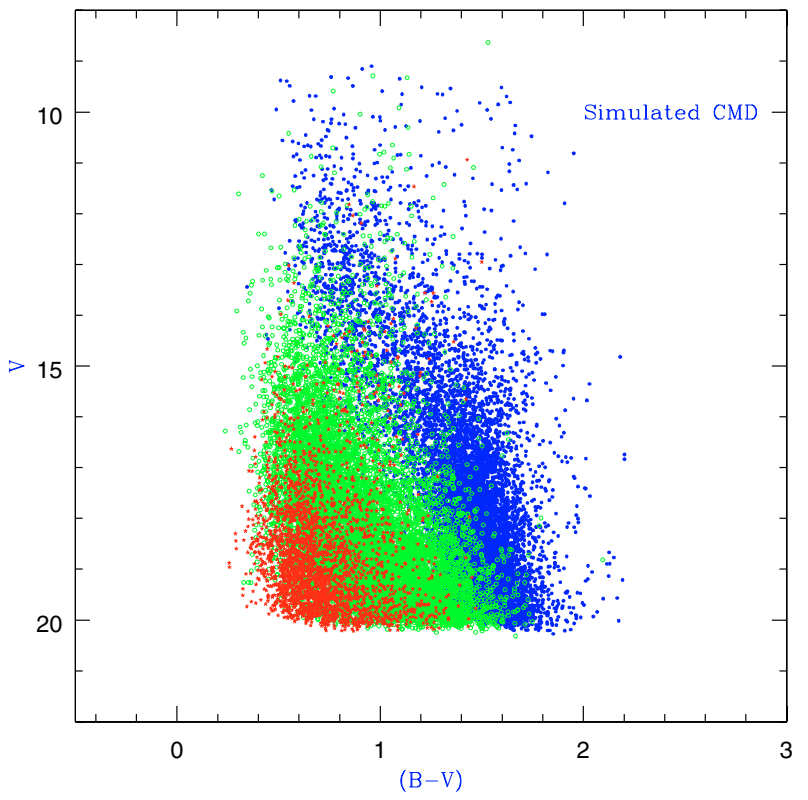

Fig. 7. Simulated CMD where different populations are shown. Black dots indicate thin disk stars, dark gray dots show thick disk objects, and light grey dots present the halo population.

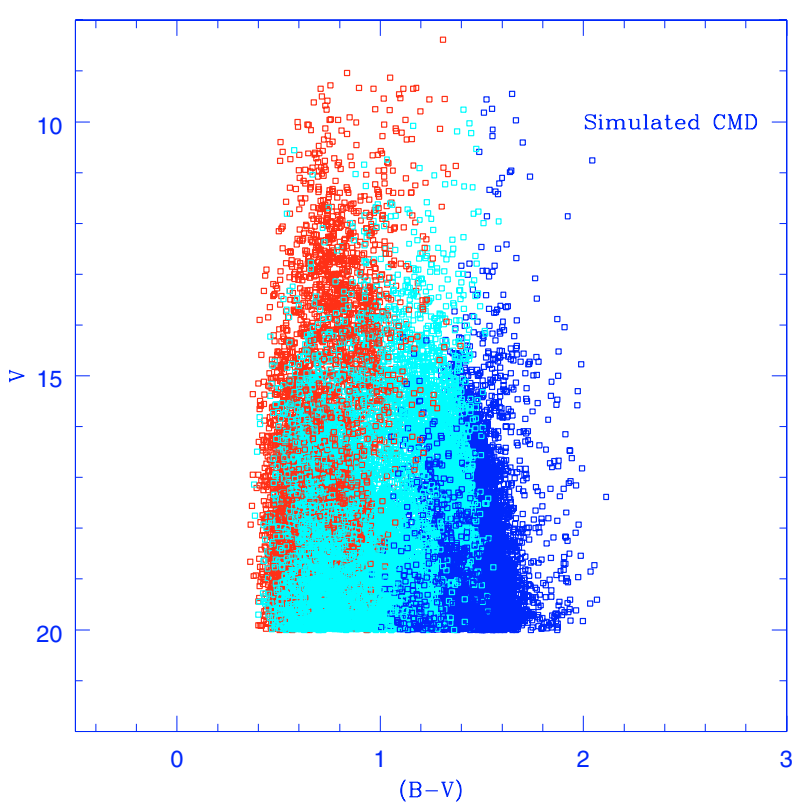

Fig. 8. Simulated CMD where different populations are shown. Black dots indicate stars having masses $<0.5 M_{\odot}$, light grey squares show stars in the mass range $0.5-0.8 M_{\odot}$, and finally dark gray squares represent stars more massive than $0.8 M_{\odot}$.

several fragmentation models (Goodwin et al. 2004; Padoan \& Nordlund 2002).

Although very reasonable, and in agreement with young cluster determinations, the present result concerning the IMF should be regarded with caution for a number of reasons:

- due to the fact that we are observing stars in a cone, we are not very sensitive to populations located very close to the Galactic plane. Concerning the thin disk, the main contribution is given by the populations older than $\sim 3$ Gyr, having a turnoff mass $<1.3 M_{\odot}$. Younger populations are underrepresented. The IMF slope we derive is referring to such a sample;

- in principle there is a dependence of the IMF on the assumed star formation rate for regions having formed stars longer than the age of the most massive stars (Binney et al. 2000; Elmegreen \& Scalo 2005). SFR variations reflect on the slope of the derived IMF. This does not apply to thick disk stars whose star formation interval is supposed to be very short in comparison to the age of the population. Stars living longer than the age of the disk will be hardly affected by the SFR changes. With an age of $10 \mathrm{Gyr}$, the turnoff mass will be of the order of $0.95 M_{\odot}$ for $Z=0.008$. For these reasons, the dependence of the results from the assumptions on the SFR is probably not extremely significant for masses lower than $0.9 M_{\odot}$, but might be more relevant at higher masses;

- kinematic effects such as stellar orbital diffusion, radial mixing by stellar waves (Binney et al. 2000) might play an important role in altering the stellar population in a given direction;

- it is clear from the previous simulations that including binary stars in the simulations plays a significant role in the determination of the IMF slope. However the percentage of binary stars in the low mass range is still poorly known (Chabrier 2003);

- stellar models for masses lower than $0.5 M_{\odot}$ suffer of uncertainties mainly regarding synthetic optical colors, the complex problem of dust formation in the coolest atmosphere models and the mass-luminosity relation (Baraffe 2002)

Table 5 gives the adopted parameters for the bulge, the ISM, the halo $\left(q_{\Phi}, v_{0}, R_{\mathrm{c}}\right)$ and the thin disk (scale lengths $\left.h_{R}\right)$ together with the parameters derived from the best fit solution (central values of the mass densities $\rho_{0}$ of the different components and scale heights) (model 7 of Table 4). The parameters related to the Thin Disk population have been tested on several Galactic fields and will be the further discussed in a forthcoming paper (Vallenari et al. 2006). Including the ISM components, the Dark Matter Halo and the WD correction of $0.027 M_{\odot} / \mathrm{pc}^{3}$ from Méndez (2002), we estimate the mass density in the solar neighborhood to be $0.06 M_{\odot} \mathrm{pc}^{-3}$. This value is in agreement with the dynamical mass estimate by Creze et al. (1998), who find $0.07 M_{\odot} \mathrm{pc}^{-3}$, whereas it is definitely lower than the local density derived by Bahcall (1984b,c); Bahcall \& Soneira (1984). The Thick Disk comes out to amount to $5 \%$ of the Thin Disk. Figure 10 gives the percentage of stars of different populations as a function of the magnitude for the model (7) of Table 4.

\subsection{Kinematics of the populations}

The best fit distribution of the proper motions is shown in Fig. 11.

Table 6 presents the best-fit determination of the velocity ellipsoids and of the circular velocity of the populations indicated in Table 6. 


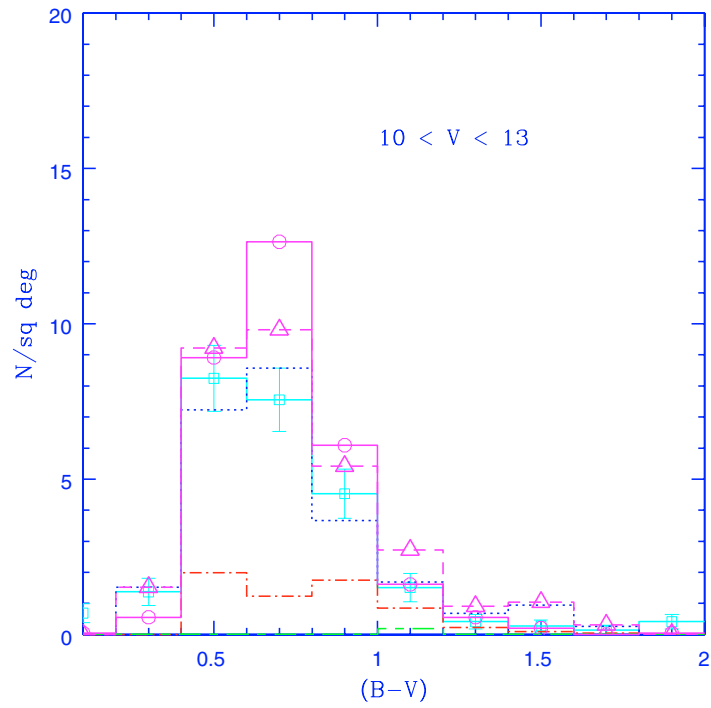

(a)

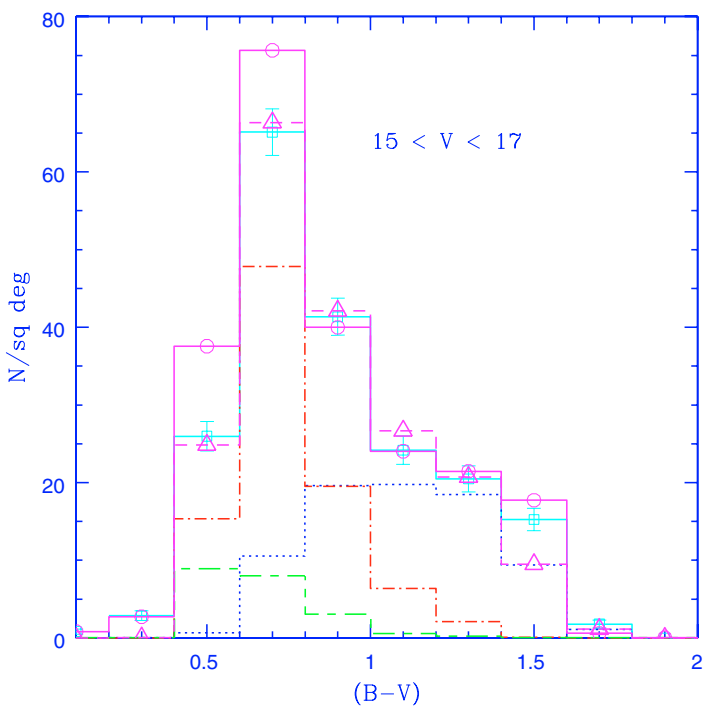

(c)

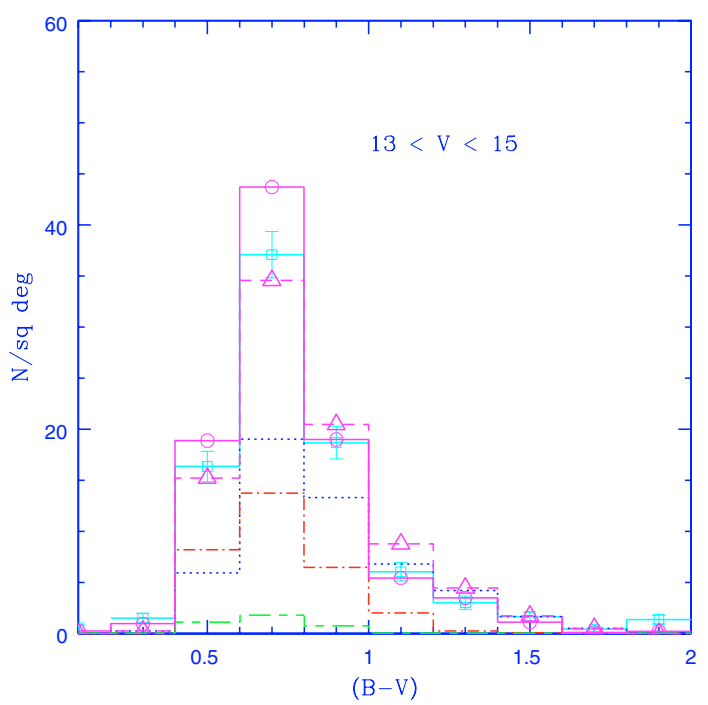

(b)

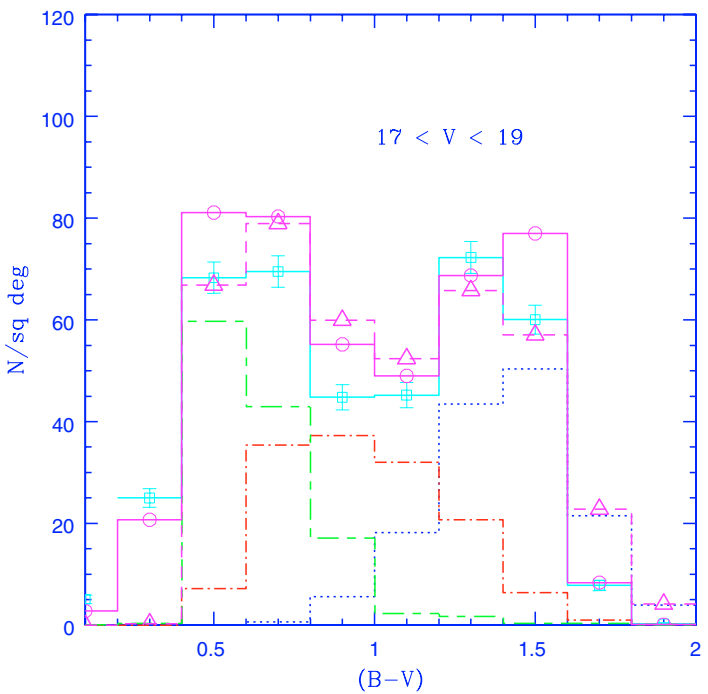

(d)

Fig. 9. Comparison of the observational and simulated colour distribution in different magnitude ranges for the model (8) of Table 3. The solid line with error-bars and squares represents the data of field F2, the solid line with circles gives the data of F3, the long-short dashed line indicates the halo stars, the dashed-dotted line shows thick disk stars, the dotted line represents the thin disk population. Finally dashed line with triangles is the total simulated LF (see text for details).

\subsubsection{Thin disk kinematics}

Since more than $80 \%$ of the Thin Disk population has ages between 3 and $10 \mathrm{Gyr}$, only velocity ellipsoid values corresponding to these populations have been derived from the data. For the population having ages younger than $3 \mathrm{Gyr}$, the values given in Table 6 are assumed. The velocity ellipsoids obtained are in good agreement with Hipparcos measurements (Gomez et al. 1997; Bienaymé 1999; Bienaymé et al. 1999).

\subsubsection{Thick disk kinematics}

From the proper motion distribution the Thick Disk is found to have a moderate rotational lag of $42 \pm 7 \mathrm{~km} \mathrm{~s}^{-1}$ with respect to the solar motion, a value comparable to the canonical $V_{\text {lag }}=50 \mathrm{~km} \mathrm{~s}^{-1}$ (Gilmore et al. 2002). In particular we find that the thin and the thick disk are well separated in the kinematic properties. The velocity ellipsoids given in Table 6 are in substantial agreement with recent determinations based on 3D-velocity analysis by Alcobé \& Cubarsi (2005); Soubiran \& Girard (2005); Soubiran et al. (2003). Different results are presented in literature. Layden et al. (1996) on the basis of field RR Lyrae survey obtain $\left(\sigma_{R R}, \sigma_{\phi \phi}, \sigma_{z z}\right)=(56 \pm 8,51 \pm 8,31 \pm 5) \mathrm{km} \mathrm{s}^{-1}$. Chiba $\&$ Beers (2001) derive from a sample of solar-neighborhood metal poor stars the velocity ellipsoid $\left(\sigma_{R R}, \sigma_{\phi \phi}, \sigma_{z z}\right)=$ $(46 \pm 4,50 \pm 4,35 \pm 5) \mathrm{km} \mathrm{s}^{-1}$. Our data are not consistent with a vertical gradient in the rotational velocity in the Thick Disk. 
Table 5. Best fit parameters (mass parameters, scale length and scale height of the Galactic components), see appendix for details.

\begin{tabular}{cccc}
\hline \hline Thin disk 1-3 Gyr & Thin disk 3-5 Gyr & Thin disk 5-7 Gyr & Thin disk 7-10 Gyr \\
\hline$h_{R}=2.5 \mathrm{kpc}$ & $h_{R}=2.5 \mathrm{kpc}$ & $h_{R}=2.5 \mathrm{kpc}$ & $h_{R}=2.5 \mathrm{kpc}$ \\
$h_{z}=0.20 \mathrm{kpc}$ & $h_{z}=0.25 \mathrm{kpc}$ & $h_{z}=0.28 \mathrm{kpc}$ & $h_{z}=0.35 \mathrm{kpc}$ \\
$\rho_{0}=2.2 \times 10^{8}\left[M_{\odot} \mathrm{kpc}^{-3}\right]$ & $\rho_{0}=1.0 \times 10^{8}\left[M_{\odot} \mathrm{kpc}^{-3}\right]$ & $\rho_{0}=2.1 \times 10^{8}\left[M_{\odot} \mathrm{kpc}^{-3}\right]$ & $\rho_{0}=8.0 \times 10^{8}\left[M_{\odot} \mathrm{kpc}^{-3}\right]$ \\
\hline Thick disk & ISM & Bulge & Halo \\
\hline$h_{R}=2.5 \mathrm{kpc}$ & $h_{R}=4.5 \mathrm{kpc}$ & $M_{\mathrm{bulge}}=3.4 \times 10^{10} M_{\odot}$ & $v_{0}=165 \mathrm{~km} \mathrm{~s}^{-1}$ \\
$h_{z}=0.9 \mathrm{kpc}$ & $h_{z}=0.20 \mathrm{kpc}$ & $a=0.7 \mathrm{kpc}$ & $R_{\mathrm{c}}=7 \mathrm{kpc}$ \\
$\rho_{0}=1.1 \times 10^{6}\left[M_{\odot} \mathrm{kpc}^{-3}\right]$ & $\rho_{0}=1.48 \times 10^{8}\left[M_{\odot} \mathrm{kpc}^{-3}\right]$ & & $q_{\Phi}=0.9$ \\
\hline
\end{tabular}

Table 6. The best-fit for the velocity dispersions and the circular velocities of the Galactic populations. All the values are in $\mathrm{km} \mathrm{s}^{-1}$.

\begin{tabular}{|c|c|c|c|c|}
\hline Parameters & Thin disk $1-3 \mathrm{Gyr}$ & Thin disk 3-5 Gyr & Thin disk 5-7 Gyr & Thin disk $7-10 \mathrm{Gyr}$ \\
\hline$\overline{\sigma_{R R, \odot}}$ & $25 \pm 2$ & $25 \pm 4$ & $38 \pm 4$ & $34 \pm 4$ \\
\hline$\sigma_{\phi \phi, \odot}$ & $20 \pm 4$ & $22 \pm 2$ & $32 \pm 2$ & $21 \pm 2$ \\
\hline \multirow[t]{2}{*}{$\sigma_{z z, \odot}$} & $10 \pm 1$ & $15 \pm 1$ & $18 \pm 1$ & $13 \pm 1$ \\
\hline & Thick disk & Halo & & \\
\hline$\sigma_{R R, \odot}$ & $74 \pm 11$ & $151 \pm 14$ & & \\
\hline$\sigma_{\phi \phi, \odot}$ & $50 \pm 7$ & $116 \pm 11$ & & \\
\hline$\sigma_{z z, \odot}$ & $38 \pm 7$ & $96 \pm 11$ & & \\
\hline$\overline{\boldsymbol{V}}_{\mathrm{c}}$ & $178 \pm 7$ & $6 \pm 19$ & & \\
\hline
\end{tabular}

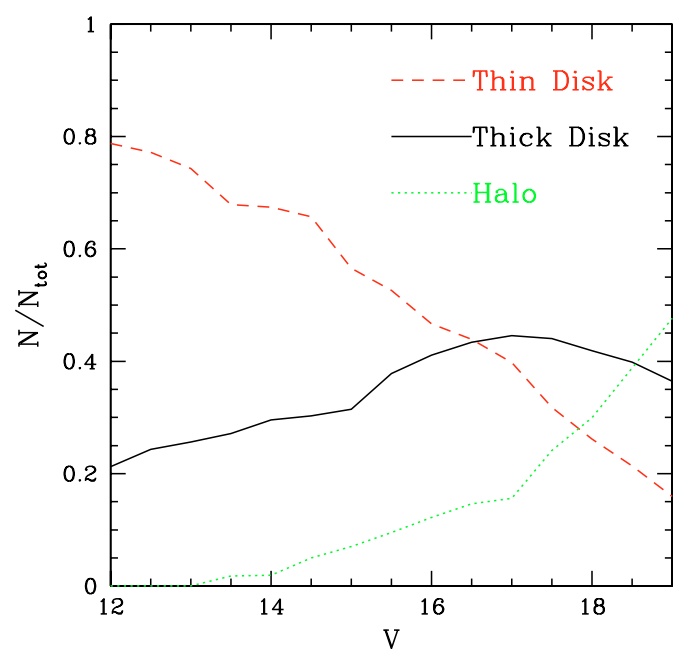

Fig. 10. Percentage of stars of different populations as a function of the magnitude for the model (7).

The presence of a gradient in the Thick Disk velocity dispersion or a multi-component structure is indicative of the formation process. Our results support the idea that the Thick Disk is formed by quick heating of the precursor Thin Disk. From the observational point of view, the situation is far from being clear. No Thick Disk velocity gradient has been detected by Soubiran (1993); Ojha et al. (1994); Soubiran et al. (2003). This suggests a formation of the Thick disk by a quick heating of the precursor Disk. Chiba \& Beers (2000) find that far from the plane, the Thick Disk has lower rotational velocity and higher velocity dispersion than close to the Galactic plane. These data are interpreted as a vertical gradient of about $30 \mathrm{~km} \mathrm{~s}^{-1} \mathrm{kpc}^{-1}$. Gilmore et al. (2002) propose a different interpretation of the data: the
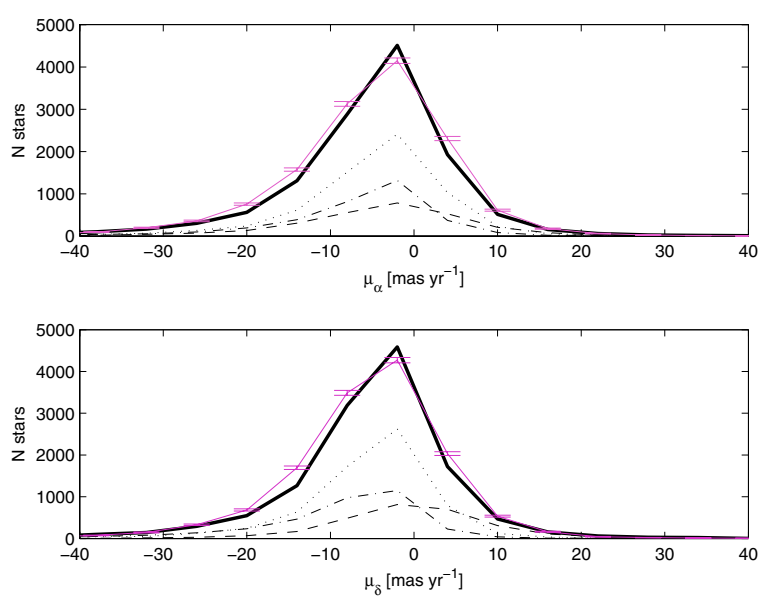

Fig. 11. The observational proper motion distribution is compared with simulated data. The heavy solid line indicates the data. The longdashed line shows the thin disk, the dot-dashed line represents the halo, and the dotted line shows the thick disk, the thin solid line gives the total simulated proper motion. Error bars are calculated using Monte Carlo simulations taking into account the observational data error (see text for details).

Thick Disk has a composite form, indicating, at some locations in the sky, the presence of relics of destroyed satellites. This can show the same effect as the presence of a vertical velocity gradient.

\subsubsection{Halo kinematics}

The Halo is characterized by $\left(\sigma_{R R}, \sigma_{\phi \phi}, \sigma_{z z}\right)=(151 \pm$ $14, \quad 116 \pm 11,96 \pm 11) \mathrm{km} \mathrm{s}^{-1}$ without any significant 
rotational velocity component for stars brighter than $V=$ 19, corresponding to a distance from the Galactic plane of about 7 kpc. Gilmore et al. (1989) and Robin \& Oblak (1987) find Halo velocity dispersions $\left(\sigma_{R R}, \sigma_{\phi \phi}, \sigma_{z z}\right)=$ $(135 \pm 20,95 \pm 10,95 \pm 10) \mathrm{km} \mathrm{s}^{-1}$ with $v_{\text {lag }}=220 \mathrm{~km} \mathrm{~s}^{-1}$. More recent determinations of the velocity ellipsoid of the Halo are by Layden et al. (1996) who find $\left(\sigma_{R R}, \sigma_{\phi \phi}, \sigma_{z z}\right)=$ $(168 \pm 13,102 \pm 8,97 \pm 7) \mathrm{km} \mathrm{s}^{-1}$ for a sample of RR Lyrae of mean metallicity $[\mathrm{Fe} / \mathrm{H}]=-1.6$. Chiba \& Beers (2000) derive $\left(\sigma_{R R}, \sigma_{\phi \phi}, \sigma_{z z}\right)=(141 \pm 11,106 \pm 9,94 \pm 8) \mathrm{km} \mathrm{s}^{-1}$ using metal poor solar neighborhood stars. The majority of the authors suggests that the Halo has little or no net rotation. However, a Halo presenting a prograde rotation is found by (Majewski et al. 1991, 1994; Majewski \& Siegel 2002) who determine $v_{\text {lag }}=280 \mathrm{~km} \mathrm{~s}^{-1}$ and Chiba \& Beers (2000) who derive $v_{\text {lag }}=250 \div 270 \mathrm{~km} \mathrm{~s}^{-1}$. These authors find that the amplitude of the prograde rotation is decreasing with increasing distance from the Galactic plane. Finally, in our data no evidence is found of the Sagittarius dwarf galaxy velocity stream down to $V=19$ (corresponding to a distance of $7 \mathrm{kpc}$ ). $N$-Body simulations of the Sagittarius orbit predict the presence of a substantial stream in this direction (Helmi \& White 2001), but at higher distances from the Galactic plane.

\section{Conclusions}

In this paper we present an update of the Padova Galaxy Model in order to simulate in a consistent way the color-magnitude diagrams and the velocity space of the Galactic populations. Particular care is paid to simulate the kinematics of the thin disk, using a velocity dispersion tensor with off-diagonal term accounting for the vertical tilt.

The model is applied to derive the stellar population properties and the kinematics of two fields in the direction of the NGP taken from GSPC-II Catalog data.

Concerning the stellar populations, one of the most critical parameters is the IMF slope. A poor fit of the color distributions at different magnitudes is obtained using a standard Kroupa IMF. The most convincing solutions are obtained when about $40 \%$ of the stars are in binaries and when the initial mass function changes slope at $m_{\mathrm{c}}=0.8 M_{\odot}$ instead than at the canonical value of $0.5 M_{\odot}$. We derive a Thin Disk IMF slope of $\alpha=0.9 \pm 0.2$ in the mass range $0.2-0.8 M_{\odot}$. The Thick Disk scale height is $h_{z \text {,thick }}=0.9 \pm 0.1 \mathrm{kpc}$.

Kinematic properties of the Disks and Halo are discussed, deriving the velocity ellipsoids, and the velocity lags. The Thick Disk is found to have a moderate rotational lag with respect to the solar motion. Its velocity ellipsoid axes are $\left(\sigma_{R R}, \sigma_{\phi \phi}, \sigma_{z z}\right)=(74 \pm 11,50 \pm 7,38 \pm 7) \mathrm{km} \mathrm{s}^{-1}$ in substantial agreement with Soubiran et al. (2003). No significant velocity gradient is found in the Thick Disk thus suggesting quick heating of the precursor disk. The Halo is characterized by the velocity ellipsoid $\left(\sigma_{R R}, \sigma_{\phi \phi}, \sigma_{z z}\right)=(151 \pm 14,116 \pm 11$, $96 \pm 11$ ), without significant rotational velocity for stars brighter than $V=18$, corresponding to a distance from the Galactic plane of about $7 \mathrm{kpc}$. In particular, the data are not consistent with a retrograde Halo rotation up to the above distance from the plane.

Acknowledgements. We are pleased to acknowledge R.L. Smart for his valuable support to this study. The Guide Star Catalog II is a joint project of the Space Telescope Science Institute and the Osservatorio Astronomico di Torino. This work was supported in part by funds from the Italian MIUR through COFIN 2001.

\section{References}

Alcobé, S., \& Cubarsi, R. 2005, A\&A, 442, 929

Amendt, P., \& Cuddeford, P. 1991, ApJ, 368, 79

Aoki, S. 1965, PASJ, 17, 273

Aoki, S. 1985, A\&A, 148, 1

Bahcall, J. N. 1984a, ApJ, 287, 926

Bahcall, J. N. 1984b, ApJ, 287, 926

Bahcall, J. N. 1984c, BAAS, 16, 733

Bahcall, J. N., \& Soneira, R. M. 1984, ApJS, 55, 67

Bania, T. M., \& Lockman, F. J. 1984, ApJS, 54, 513

Baraffe, I. 2002, in EAS Publications Series, 191

Baraffe, I., Chabrier, G., Allard, F., \& Hauschildt, P. H. 1998, A\&A, 337,403

Beers, T. C., \& Sommer-Larsen, J. 1995, ApJS, 96, 175

Beers, T. C., Chiba, M., Yoshii, Y., et al. 2000, AJ, 119, 2866

Bertelli, G., Bressan, A., Chiosi, C., Ng, Y. K., \& Ortolani, S. 1995, A\&A, 301, 381

Bienaymé, O. 1999, A\&A, 341, 86

Bienayme, O., Robin, A. C., \& Creze, M. 1987, A\&A, 180, 94

Bienaymé, O., Crézé, M., Chereul, E., \& Pichon, C. 1999, in Galaxy Dynamics - A Rutgers Symposium, ASP Conf. Ser., 182, 301

Binney, J., \& Merrifield, M. 1998, Galactic astronomy (Princeton, NJ: Princeton University Press)

Binney, J., \& Tremaine, S. 1994, Galactic Dynamics (Princeton, NJ: Princeton University Press)

Binney, J., Dehnen, W., \& Bertelli, G. 2000, MNRAS, 318, 658

Brand, J., \& Blitz, L. 1993, A\&A, 275, 67

Briceño, C., Luhman, K. L., Hartmann, L., Stauffer, J. R., \& Kirkpatrick, J. D. 2002, ApJ, 580, 317

Bucciarelli, B., García Yus, J., Casalegno, R., et al. 2001, A\&A, 368, 335

Carlberg, R. G., \& Sellwood, J. A. 1985, ApJ, 292, 79

Carlberg, R. G., Dawson, P. C., Hsu, T., \& Vandenberg, D. A. 1985, ApJ, 294, 674

Chabrier, G. 2003, PASP, 115, 763

Chiba, M., \& Beers, T. C. 2000, AJ, 119, 2843

Chiba, M., \& Beers, T. C. 2001, ApJ, 549, 325

Combes, F. 1999, in Galaxy Evolution: Connecting the Distant Universe with the Local Fossil Record, 417

Creze, M., Chereul, E., Bienayme, O., \& Pichon, C. 1998, A\&A, 329, 920

Dehnen, W., \& Binney, J. 1998a, MNRAS, 294, 429

Dehnen, W., \& Binney, J. J. 1998b, MNRAS, 298, 387

Delhaye, J. 1965, Galactic Structure, ed. A. Blaauw, \& M. Schmidt (Chicago University Press)

Drimmel, R., \& Spergel, D. N. 2001, ApJ, 556, 181

Duquennoy, A., \& Mayor, M. 1991, A\&A, 248, 485

Duquennoy, A., Mayor, M., \& Halbwachs, J.-L. 1991, A\&AS, 88, 281

Efron, B. 1982, The Jackknife, the Bootstrap and other resampling plans, CBMS-NSF Regional Conference Series in Applied Mathematics (Philadelphia: Society for Industrial and Applied Mathematics (SIAM))

Elmegreen, B. G., \& Scalo, J. 2005, ApJ, 636, 149 
Famaey, B., Jorissen, A., Luri, X., et al. 2005, A\&A, 430, 165

Fischer, D. A., \& Marcy, G. W. 1992, ApJ, 396, 178

Freeman, K., \& Bland-Hawthorn, J. 2002, ARA\&A, 40, 487

Fricke, W. 1952, Astron. Nachr., 280, 193

Fuchs, B., \& Wielen, R. 1987, in The Galaxy, NATO ASIC Proc., 207, 375

Fuchs, B., Dettbarn, C., Jahreiß, H., \& Wielen, R. 2001, in Dynamics of Star Clusters and the Milky Way, ASP Conf. Ser., 228, 235

Gómez, A. E., Grenier, S., Udry, S., et al. 1998, Highlights in Astronomy, 11, 574

Gilmore, G., Wyse, R. F. G., \& Kuijken, K. 1989, ARA\&A, 27, 555

Gilmore, G., Wyse, R. F. G., \& Norris, J. E. 2002, ApJ, 574, L39

Girardi, L., Bressan, A., Bertelli, G., \& Chiosi, C. 2000, A\&AS, 141, 371

Gomez, A. E., Grenier, S., Udry, S., et al. 1997, in ESA SP-402: Hipparcos - Venice '97, 621

Goodwin, S. P., Whitworth, A. P., \& Ward-Thompson, D. 2004, A\&A, 419, 543

Helmi, A., \& de Zeeuw, P. 2000, MNRAS, 319, 657

Helmi, A., \& White, S. D. M. 2001, MNRAS, 323, 529

Hernquist, L. 1990, ApJ, 356, 359

Hill, G., Hilditch, R. W., \& Barnes, J. V. 1979, MNRAS, 186, 813

Holtzman, J. A., Gallagher, J. S., Cole, A. A., et al. 1999, AJ, 118, 2262

Hunter, C. 1975, AJ, 80, 783

Hunter, C. 1979, ApJ, 227, 73

Kerr, F. J., \& Lynden-Bell, D. 1986, MNRAS, 221, 1023

King, I. 1990, The Milky Way as Galaxy (New York, NY: University Science Books), 10012

Kinman, T. D., Saha, A., \& Pier, J. R. 2004, ApJ, 605, L25

Kinman, T. D., Bragaglia, A., Cacciari, C., Buzzoni, A., \& Spagna, A. 2005, in The Three-Dimensional Universe with Gaia, ESA SP-576, 175

Knapp, G. R., \& Morris, M. 1985, ApJ, 292, 640

Knude, J., Winther, M., \& Schnedler Nielsen, H. 1987, A\&A, 179, 115

Kochanek, C. S. 1996, ApJ, 457, 228

Kroupa, P. 2001a, MNRAS, 322, 231

Kroupa, P. 2001b, in Dynamics of Star Clusters and the Milky Way, ASP Conf. Ser., 228, 187

Kuijken, K., \& Gilmore, G. 1989a, MNRAS, 239, 651

Kuijken, K., \& Gilmore, G. 1989b, MNRAS, 239, 605

Kuijken, K., \& Gilmore, G. 1989c, MNRAS, 239, 571

Lada, C. J., \& Lada, E. A. 2003, ARA\&A, 41, 57

Larsen, J. A., \& Humphreys, R. M. 2003, AJ, 125, 1958

Layden, A. C., Hanson, R. B., Hawley, S. L., Klemola, A. R., \& Hanley, C. J. 1996, AJ, 112, 2110

Lewis, J. R., \& Freeman, K. C. 1989, AJ, 97, 139

Lin, D. N. C., Jones, B. F., \& Klemola, A. R. 1995, ApJ, 439, 652

Luhman, K. L. 2004, ApJ, 617, 1216

Luhman, K. L., \& Potter, D. 2006, ApJ, 638, 887

Luhman, K. L., Briceño, C., Stauffer, J. R., et al. 2003, ApJ, 590, 348

Méndez, R. A. 2002, A\&A, 395, 779

Méndez, R. A., Platais, I., Girard, T. M., Kozhurina-Platais, V., \& van Altena, W. F. 2000, AJ, 119, 813

Majewski, S. R. 1993, ARA\&A, 31, 575

Majewski, S. R., \& Siegel, M. H. 2002, ApJ, 569, 432

Majewski, S. R., Munn, J. A., Kron, R. G., et al. 1991, in The Space Distribution of Quasars, ASP Conf. Ser., 21, 55

Majewski, S. R., Munn, J. A., \& Hawley, S. L. 1994, ApJ, 427, L37

Mason, B. D., Henry, T. J., Hartkopf, W. I., Ten Brummelaar, T., \& Soderblom, D. R. 1998, AJ, 116, 2975
Mayor, M. 1974, A\&A, 32, 321

Mendez, R. A., \& van Altena, W. F. 1996, AJ, 112, 655

Merritt, D. 1985a, MNRAS, 214, 25P

Merritt, D. 1985b, AJ, 90, 1027

Meusinger, H., Stecklum, B., \& Reimann, H.-G. 1991, A\&A, 245, 57 Michie, R. W. 1963, MNRAS, 125, 127

Muench, A. A., Lada, E. A., Lada, C. J., et al. 2003, AJ, 125, 2029

Nelder, J., \& Mead, R. 1965, Computer J., 7, 308

Ng, Y. K., Bertelli, G., Chiosi, C., \& Bressan, A. 1997, A\&A, 324, 65

Nordström, B., Mayor, M., Andersen, J., et al. 2004, A\&A, 418, 989

Ojha, D. K., Bienayme, O., Robin, A. C., \& Mohan, V. 1994, A\&A, 290,771

Olling, R. P., \& Merrifield, M. R. 1998, MNRAS, 297, 943

Osipkov, L. P. 1979, Pis'ma v Astronomicheskii Zhurnal, Feb. 1979, 5, 77, Soviet Astronomy Letters, Jan.-Feb. 1979, 5, 42, Translation, in Russian, 5, 77

Padoan, P., \& Nordlund, Å. 2002, ApJ, 576, 870

Pasetto, S. 2005, Ph.D. Thesis, University of Padova, Italy

Pont, F., Queloz, D., Bratschi, P., \& Mayor, M. 1997, A\&A, 318, 416

Quinn, P. J., \& Goodman, J. 1986, ApJ, 309, 472

Ratnatunga, K. U., Bahcall, J. N., \& Casertano, S. 1989, ApJ, 339, 106

Reylé, C., \& Robin, A. C. 2002, Ap\&SS, 281, 115

Robin, A. C., \& Oblak, E. 1987, in European Regional Astronomy Meeting of the IAU, 4, 323

Robin, A. C., Reylé, C., Derrière, S., \& Picaud, S. 2003, A\&A, 409, 523

Rocha-Pinto, H. J., Flynn, C., Scalo, J., et al. 2004, A\&A, 423, 517

Sakamoto, T., Chiba, M., \& Beers, T. C. 2003, A\&A, 397, 899

Sawa, T., Fujimoto, M., \& Kumai, Y. 1999, in New Views of the Magellanic Clouds, IAU Symp., 190, 499

Schultheis, M., Robin, A. C., Reylé, C., et al. 2005, [arXiv: astro-ph/0510083]

Schwarzschild, K. 1907, Gotting Nach.

Sellwood, J. A., \& Binney, J. J. 2002, MNRAS, 336, 785

Shu, F. H. 1969, ApJ, 158, 505

Soubiran, C. 1993, A\&A, 274, 181

Soubiran, C., \& Girard, P. 2005, A\&A, 438, 139

Soubiran, C., Bienaymé, O., \& Siebert, A. 2003, A\&A, 398, 141

Spagna, A., Bucciarelli, B., Carollo, D., et al. 2005, in The ThreeDimensional Universe with Gaia, ESA SP-576, 193

Stromgren, B. 1987, in The Galaxy, NATO ASIC Proc. 207, 229

Trumpler, R. J., \& Weaver, H. F. 1953, Statistical astronomy (New York: Dover Publications, Dover Books on Astronomy and Space Topics)

Vallenari, A., Bertelli, G., \& Schmidtobreick, L. 2000, A\&A, 361, 73

Vallenari, A., Ragaini, S., \& Bertelli, G. 2006, in preparation

van der Kruit, P. C. 1988, A\&A, 192, 117

van der Kruit, P. C., \& Searle, L. 1982, A\&A, 110, 61

Vandervoort, P. O. 1975, ApJ, 195, 333

von Seeliger, H. 1898, Abh. Bayerische Akad. Wiss., Math.-Phys. KI, 19,564

Wainscoat, R. J., Freeman, K. C., \& Hyland, A. R. 1989, ApJ, 337, 163

Weaver, H., \& Williams, D. R. W. 1973, A\&AS, 8, 1

Weaver, H., \& Williams, D. R. W. 1974a, A\&AS, 17, 1

Weaver, H., \& Williams, D. R. W. 1974b, A\&AS, 17, 251

Wielen, R. 1974a, PASP, 86, 341

Wielen, R. 1974b, A\&AS, 15, 1

Wielen, R., Dettbarn, C., Fuchs, B., Jahreiß, H., \& Radons, G. 1992, in The Stellar Populations of Galaxies, IAU Symp., 149, 81 
A. Vallenari et al.: Milky Way Populations toward the North Galactic Pole, Online Material p 1

\section{Online Material}




\section{Appendix}

In this section, more detail is given about the adopted Potential. The predictions of the kinematic model such as rotation curve, Oort functions, terminal velocity, vertical force on the plane, total mass are derived and discussed. Finally, the predicted vertical tilt angle of the thin disk velocity ellipsoid is shown (Pasetto 2005, more detail can be found in).

\section{A.1. The potential}

We assume:

a) a logarithmic profile for the dark matter and stellar halo profile from as in Helmi \& de Zeeuw (2000):

$\Phi_{\text {halo }}(R, z)=\frac{v_{0}^{2}}{2} \ln \left(R_{\mathrm{c}}^{2}+R^{2}+\frac{z^{2}}{q_{\Phi}^{2}}\right)+$ const.

with $v_{0}^{2}=165 \mathrm{~km} \mathrm{~s}^{-1}$ (see Table 5) where $v_{0}$ is the velocity-scale for the halo rotation curve, $R_{\mathrm{c}}=6 \mathrm{kpc}$ is the length scale and $q_{\Phi} \leq 1$ is the ellipticity control parameter for the potential isocontour (see Binney \& Tremaine 1994);

b) an Hernquist (1990) profile for the Bulge:

$\Phi_{\text {bulge }}=-\frac{G M_{\text {bulge }}}{r+a}$

where $M_{\text {bulge }}$ is the Bulge mass, and a is the scale length $0.7 \mathrm{kpc}$ long;

c) the $k$ disk component density is described by the double exponential law:

$\rho_{\text {disk }(\mathrm{k})}(R, z)=\rho_{C(k)} \exp \left(-\frac{R}{h_{R(k)}}-\frac{|z|}{h_{z(k)}}\right)$.

From this density the potential is derived by numerical integration

$\Phi_{\operatorname{Disk}(\mathrm{k})}(R, z)=-4 \pi G \frac{\rho_{C(k)}}{h_{R(k)}} \int_{0}^{+\infty} \frac{J_{0}(x R)}{\left(h_{R(k)}^{-2}+x^{2}\right)^{3 / 2}} \frac{h_{z(k)}^{-1} \mathrm{e}^{-x|z|}-x e^{-h_{z(k)}^{-1}|z|}}{h_{z(k)}^{-2}-x^{2}} \mathrm{~d} x$

with $k=$ thin disk (young and old), thick disk, following Quinn \& Goodman (1986).

\section{A.2. The rotation curve}

Given a gravitational potential, the rotation velocity is

$v_{\mathrm{c}}=\sqrt{R \frac{\partial \Phi_{\mathrm{tot}}}{\partial R}}$

The problem is reduced to calculate the contribution from the various components of the galaxy. Here we report, as an example, only the result for the rotation curve. After simple passages one obtains:

$v_{\mathrm{c}}=\sqrt{R\left(4 G \pi R \sum_{k=1}^{n} \alpha_{k} \rho_{\mathrm{c}, k} \int_{0}^{\infty} \frac{k J_{1}(x R)}{\left(x^{2}+\alpha_{k}^{2}\right)^{3 / 2}\left(x+\beta_{k}\right)} \mathrm{d} x+\frac{R v_{0}^{2}}{R^{2}+R_{\mathrm{c}}^{2}}+\frac{3 G M_{\text {bulge }}}{(c+R)^{2}}\right)}$

with $n=4$ as above for the four disk-like components.

The rotation curve of Fig. A.1 is obtained using the parameters of Table 5. This curve is comparable with the one derived by Dehnen \& Binney (1998a) in their best-fit model.

\section{A.3. The Oort functions}

The Oort functions $A(R)$ and $B(R)$ are given by:

$$
\begin{aligned}
& A(R) \equiv \frac{1}{2}\left(\frac{v_{\mathrm{c}}(R)}{R}-\frac{\mathrm{d} v_{\mathrm{c}}(R)}{\mathrm{d} R}\right) \\
& B(R) \equiv-\frac{1}{2}\left(\frac{v_{\mathrm{c}}(R)}{R}+\frac{\mathrm{d} v_{\mathrm{c}}(R)}{\mathrm{d} R}\right) .
\end{aligned}
$$


A. Vallenari et al.: Milky Way Populations toward the North Galactic Pole, Online Material $p 3$

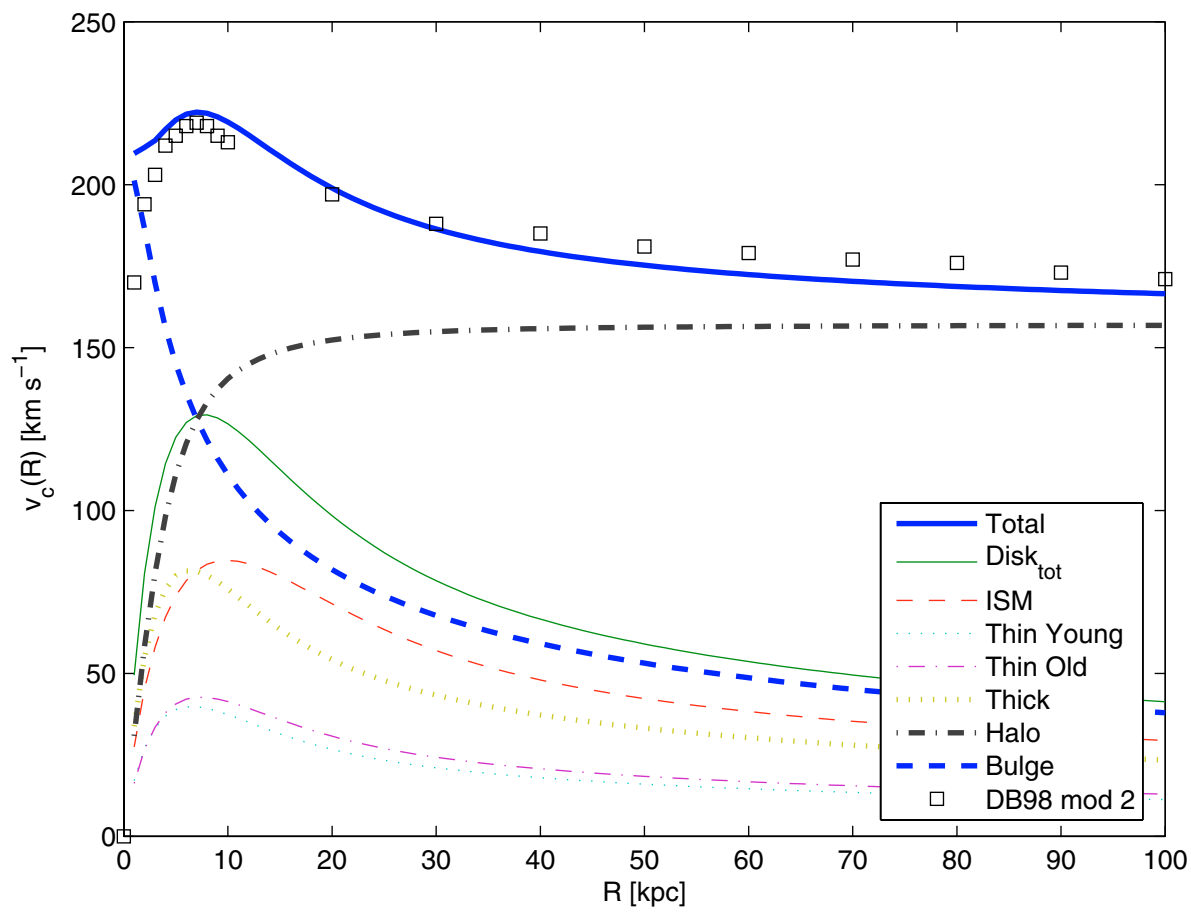

Fig. A.1. The rotation curve for each sub-component of the galaxy model. The solid bold line is the total rotational curve. The solid thin line is disk-total components: thin young disk (dotted line), old thin disk (dot-dashed line), thick disk (dotted thin line) and ISM (dashed line). Squares indicate the model of Dehnen \& Binney (1998a).

The value of these functions in the solar vicinity are the Oort constants. The quantity $B$ is determined by studying the dependence of stellar proper motions on the Galactic longitude, whereas $A$ is derived from the analysis of either proper motions or radial velocities in the solar vicinity:

$v_{r}=A d \sin 2 l$

$\mu_{l}=\frac{A \cos 2 l+B}{4.74}$

where $d$ is the distance to the object in kpc, $l$ is the Galactic longitude, $A$ and $B$ are in $\mathrm{km} \mathrm{s}^{-1} \mathrm{kpc}^{-1}$ and $\mu_{l}$ in mas $\mathrm{yr}^{-1}$.

$A-B=\frac{v_{\mathrm{c}}}{R}$ can be derived in an independent way from proper motion surveys in the direction $l=90^{\circ}$ or $l=270^{\circ}$, because along these directions the star distribution has a small dependence on the Galactocentric radius and is slightly affected by the radial dependence of the Oort functions. Finally, the combination $-\frac{B}{A-B}$ can be estimated from the velocity ellipsoid of random stellar motions. From our assumptions, we derive in the solar vicinity $A=14.2$ and $B=-13.7$, in agreement with the values $A=14.5 \pm 1.5$ and $B=-13.5 \pm 2$ proposed by Dehnen \& Binney (1998a), Olling \& Merrifield (1998). Figure A.2 shows the Oort functions, $A-B$ and $-\frac{B}{A-B}$. These functions are in agreement with the data form Olling $\&$ Merrifield (1998).

\section{A.4. The terminal velocity}

For an axisymmetric Galaxy with circular rotating interstellar medium, the peak velocity along a given line-of sight (l.o.s.) at $b=0$ and $l$ either in the first or in the fourth quadrant, occurs at the radius $R=R_{\odot} \sin l$. This terminal velocity $v_{\text {term }}$ is related to the circular speed $v_{\mathrm{c}}$ by

$v_{\text {term }}=v_{\mathrm{c}}\left(R_{\odot} \sin l\right)-v_{\mathrm{c}}(R) \cdot \sin l$.

However, the non circular motion of the ISM induced by spiral arms leads to deviation from this ideal relation (outside the region $|l| \leq 20^{\circ}$ dominated by the bar, these deviations are expected to be small). Figure A. 3 compares the terminal velocity derived by us with the model by Dehnen \& Binney (1998a). Figure A.4 shows both the direction and the value of $v_{\text {term }}$ on the galactic plane. 
A. Vallenari et al.: Milky Way Populations toward the North Galactic Pole, Online Material p 4
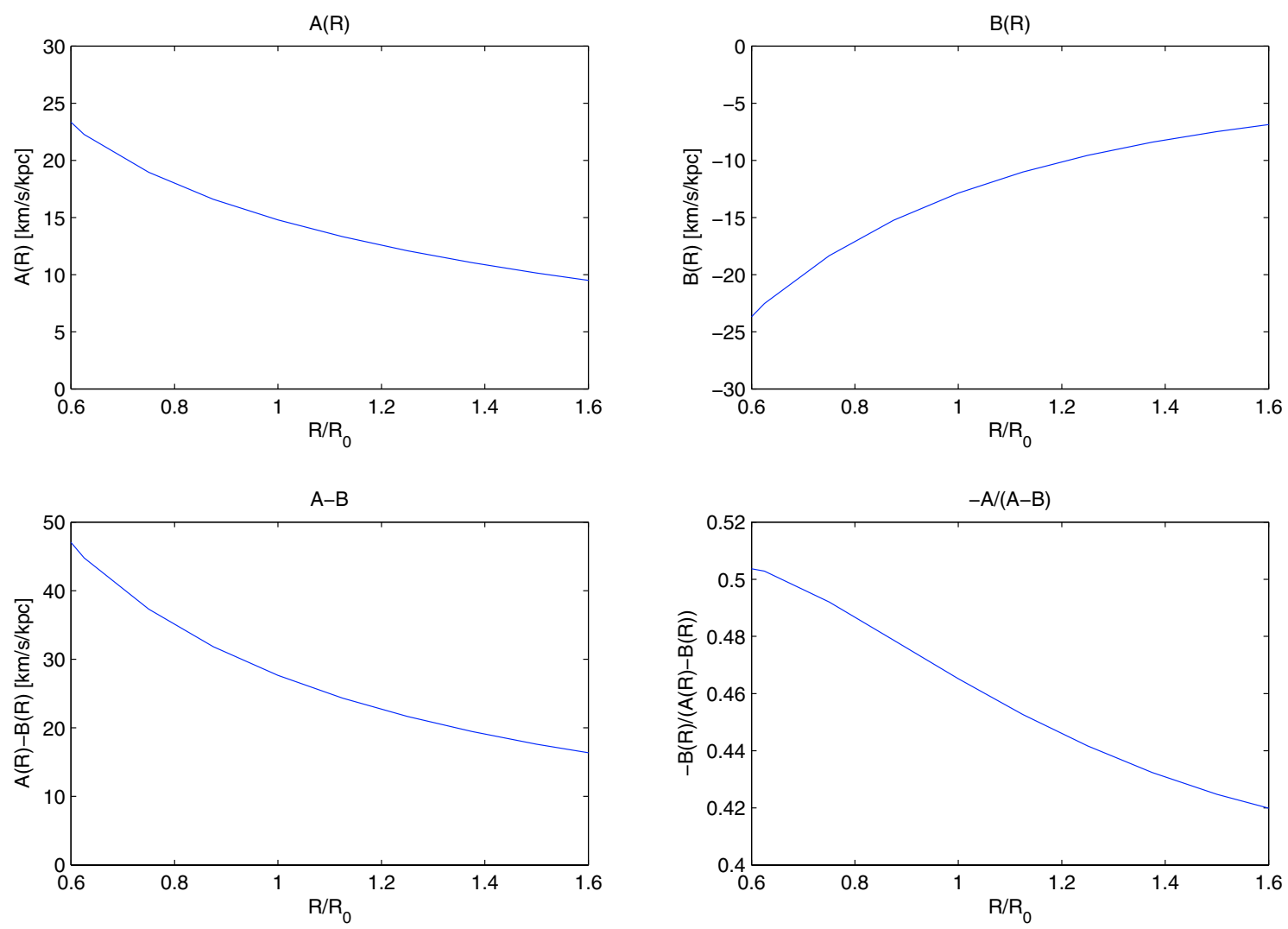

Fig. A.2. The Oort functions $A(R)$ and $B(R)$ as derived from the model of Fig. A.1 together with the values of $A-B$ and $-B /(A-B)$. The units of $A, B$ and $A-B$ are $\mathrm{km} \mathrm{s}^{-1} \mathrm{kpc}^{-1}$. The smooth behavior depends on the fact that they have been derived directly form the potential. The predicted trend given by these functions is in agreement with the observational estimates of these quantities (Olling \& Merrifield 1998, and reference therein).

\section{A.5. The rotation curve of the outer Galaxy}

For an axisymmetric galaxy, the radial velocity relative to the LSR, $v_{R, \mathrm{LSR}}$, of a circularly orbiting object at galactocentric coordinates $(l, b)$ and galactocentric radius $R$ is related to the circular velocity by

$W(R)=\frac{v_{R, \mathrm{LSR}}}{\sin l \cos b}=\frac{R_{\odot}}{R} v_{\mathrm{c}}(R)-v_{\mathrm{c}}\left(R_{\odot}\right)$.

As well known, for $R>R_{\odot}$, one cannot deduce $R$ for an object at given $l$ without a knowledge of the distance $d$. Widely used data to constrain the rotational curve of the outer galaxy are those by Brand \& Blitz (1993) who list HII regions that have spectro-photometric distances and associated molecular clouds with measured radial velocities, Pont et al. (1997) who give radial velocities and photometry for classical Cepheids (Olling \& Merrifield 1998; Dehnen \& Binney 1998a). Figure A.5 compares the values of $W$ derived from our model with the available data.

\section{A.6. The total mass inside a radius $R<100 \mathrm{Kpc}$}

The total mass within a sphere of radius $R$ can be estimated from the velocity distribution of the Milky Way's satellites (Zaho 1998), from the maximal locally observed stellar velocity and argumentation on the escape speed, from the LSR (Robin et al. 2003), from the timing of the local group (Combes 1999) and finally from the dynamics of the Magellanic Clouds and Stream (Lin et al. 1995; Sawa et al. 1999). Sakamoto et al. (2003) derived the mass reproducing the kinematics of the galactic satellites from the Halo globular clusters and the horizontal branch stars. The total mass inside the radius of $100 \mathrm{kpc}$ can be calculated integrating the density profile. The model presented above gives a mass of $M_{\mathrm{tot}}(r<100 \mathrm{kpc})=8.8 \times 10^{11} M_{\odot}$, fully compatible with the values presented in literature e.g.:

- Kochanek (1996): $M_{\text {tot }}(R<100 \mathrm{kpc})=7 \pm 2 \times 10^{11} M_{\odot}$;

- Lin et al. (1995): $M_{\text {tot }}(R<100 \mathrm{kpc})=5 \pm 2 \times 10^{11} M_{\odot}$;

- Dehnen \& Binney (1998a): $M_{\mathrm{tot}}(R<100 \mathrm{kpc})=8 \pm 2 \times 10^{11} M_{\odot}$; 


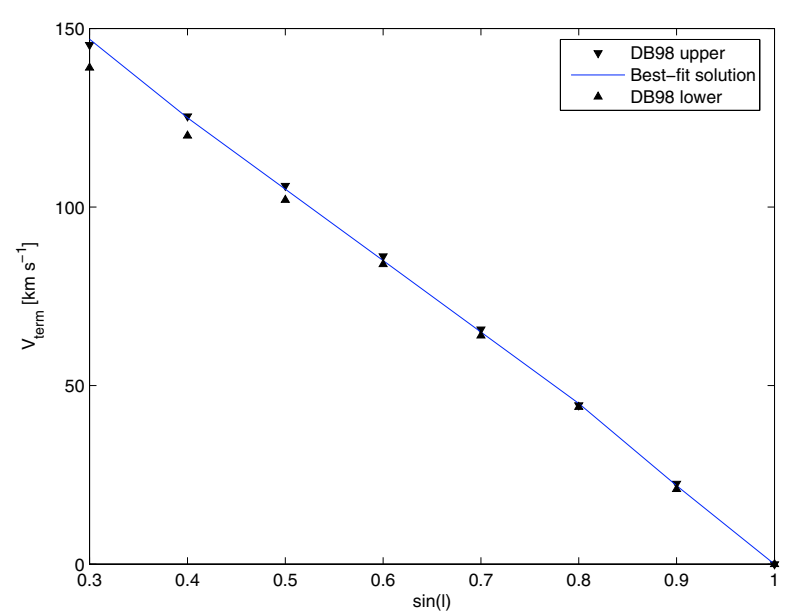

Fig. A.3. The terminal velocity derived in this work (solid line). The model is in good agreement with the model by Dehnen \& Binney (1998a) reproducing the data from Dehnen \& Binney (1998a), Brand \& Blitz (1993) and Pont et al. (1997).

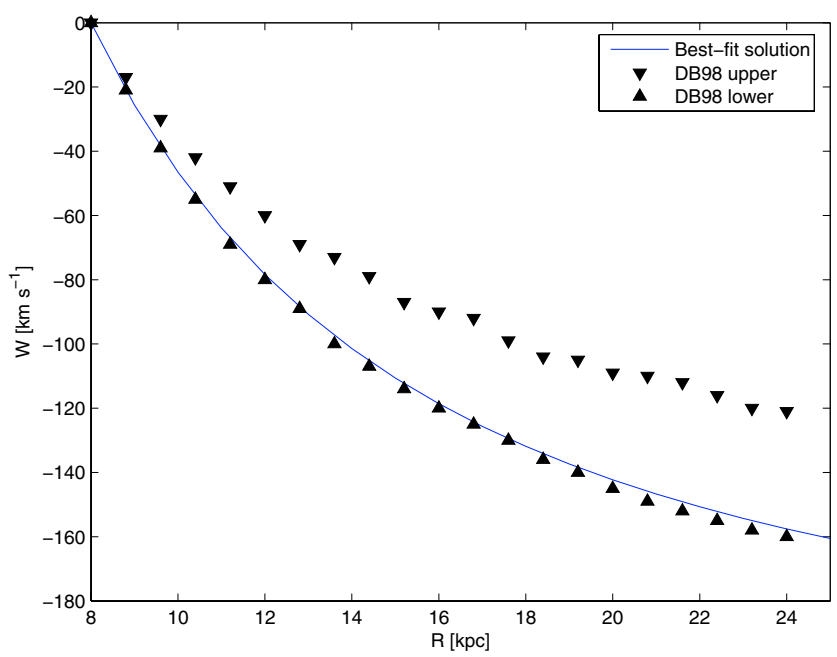

Fig. A.5. The numerical model reproduces the trend of $W(R)$ as derived by Dehnen \& Binney (1998b) in agreement with the data of Weaver \& Williams (1973, 1974a,b); Bania \& Lockman (1984); Knapp \& Morris (1985), and Kerr \& Lynden-Bell (1986). Upper and lower model from Dehnen \& Binney (1998a) are plotted.

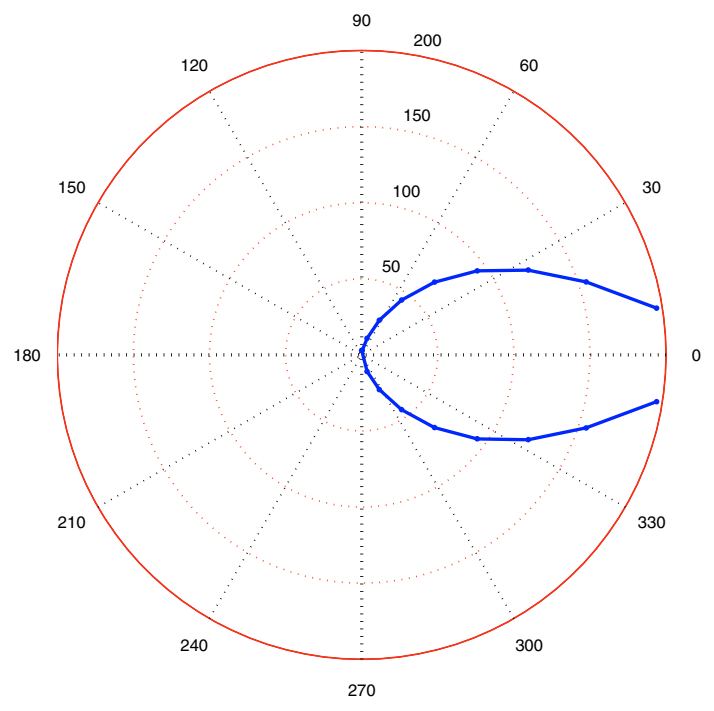

Fig. A.4. The terminal velocity as in Fig. A.3. In this plot both the direction and the value of the terminal velocity are shown at the galactic plane. The angular degree refers to the $l$ galactic longitude and the dashed rings show the $v_{\text {term }}$ levels in the specified direction. The zero direction correspond to the galactic center and the zone of avoidance near 0 is due to the presence of the bulge.

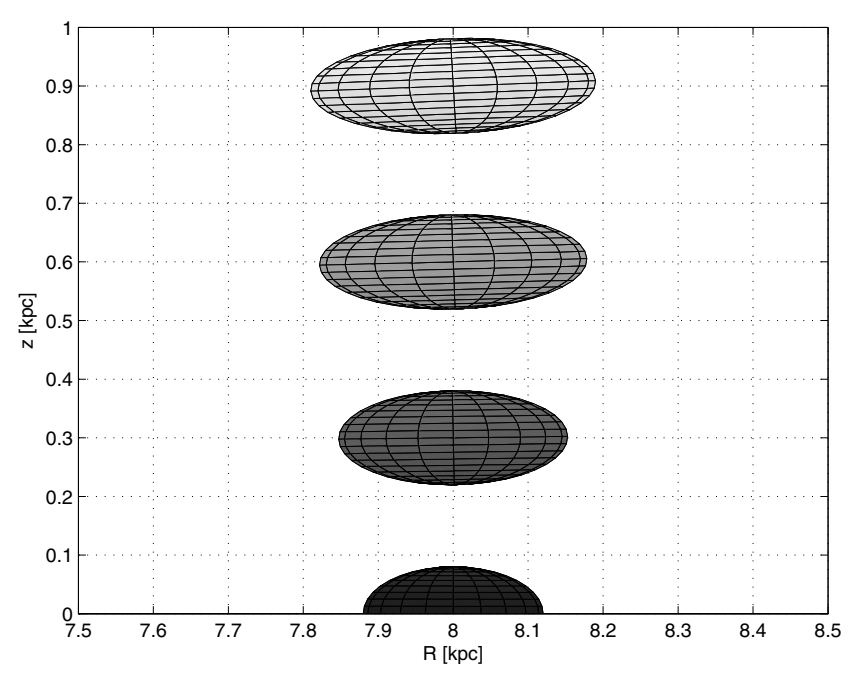

Fig. A.6. The tilt of the vertical ellipsoid is shown for the solar neighborhood. The picture shows the shape of the velocity ellipsoid of the solar position up till $z=1 \mathrm{kpc}$.

\section{A.7. The vertical force on the plane}

The gravitational force toward the galactic plane can be measured from data describing the balance between the gravitational attraction toward the plane and the pressure (i.e. the velocity dispersion) force away from the plane of a stellar "tracer" population (Kuijken \& Gilmore 1989c,b,a). The basis of the authors analysis is the standard assumption that the $z$-motion of disk stars near the sun can be described by one-dimensional Boltzmann equation. Having the vertical density and the vertical energy integral, they perform an Abel inversion to obtain the distribution in the phase space. Independently of the details of the adopted Galactic model they concluded that the force due to all the matter below $z= \pm 1.1 \mathrm{kpc}$ is

$\left|F_{z}(1.1 \mathrm{kpc})\right|=2 \pi G \cdot(71 \pm 6) M_{\odot} \mathrm{pc}^{-2}$.

Using our model of the Galactic potential, we derive:

$\left|F_{z}(1.1 \mathrm{kpc})\right|=2 \pi G \cdot(68) M_{\odot} \mathrm{pc}^{-2}$

in good agreement with the quoted estimate. 
A. Vallenari et al.: Milky Way Populations toward the North Galactic Pole, Online Material p 6

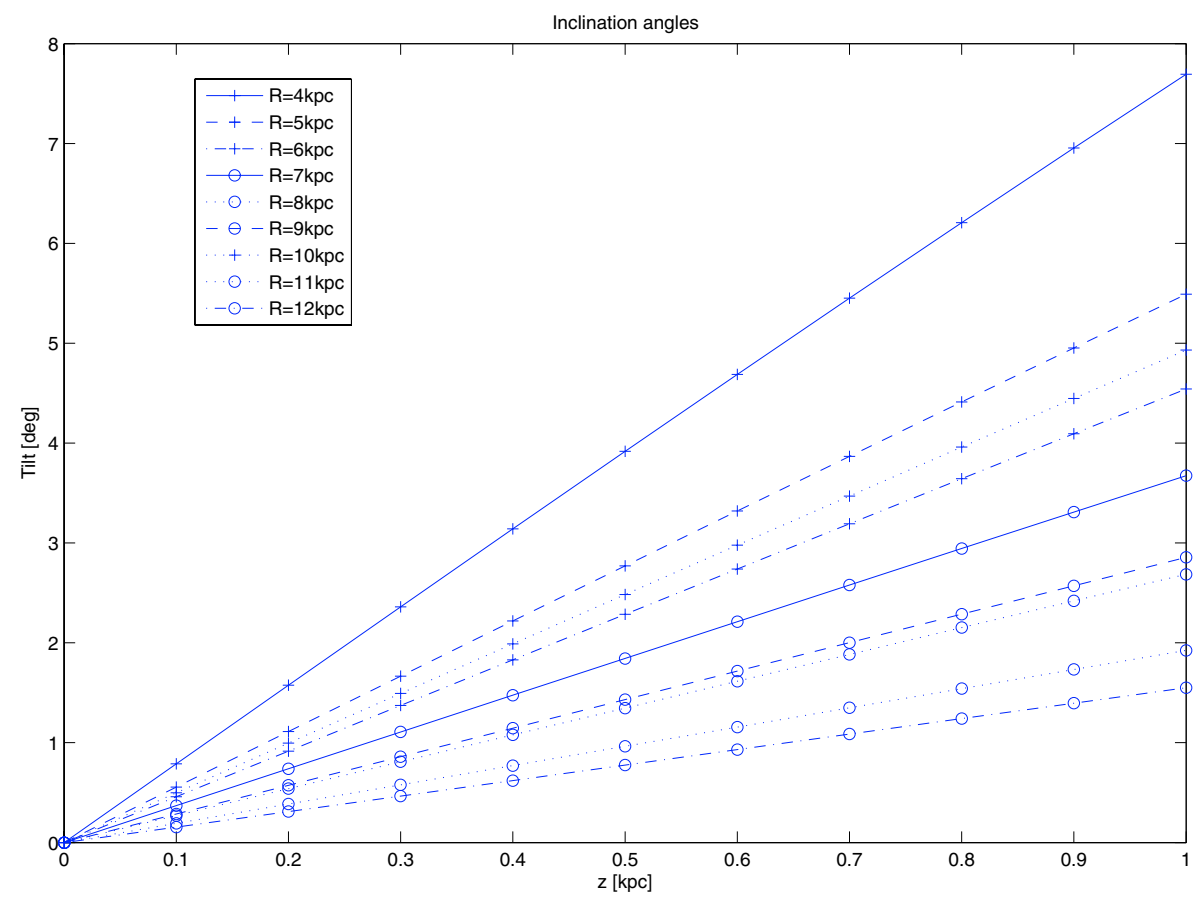

Fig. A.7. Tilt angle $\beta$ of the velocity ellipsoid for different distances from the Galactic center. The plot can be better understood in comparison with Fig. A.6.

\section{A.8. The thin disk off-diagonal term of the velocity ellipsoid}

As already described in the previous Sections, to derive the off diagonal terms $\sigma_{R z}$ for the thin disk we follow Amendt \& Cuddeford (1991) approximations:

$\lambda(R)=\left(\frac{R^{2} \Phi_{, R z z}}{3 \Phi_{, R}+R \Phi_{, R R}-4 R \Phi_{, z z}}\right)(R, z=0)$.

From the above equation and Eq. (26), the vertical tilt angle $\beta$ is derived. Figures A.6 and A.7 present the predicted tilt angle $\beta$ as a function of the height $z$ on the GP in the solar vicinity and at different radii, respectively. 